\title{
TITLE:
}

\section{A DNA-based molecular motor that can navigate a network of tracks}

\section{$\operatorname{AUTHOR}(\mathrm{S})$ :}

Wickham, Shelley F. J.; Bath, Jonathan; Katsuda, Yousuke; Endo, Masayuki; Hidaka, Kumi; Sugiyama, Hiroshi; Turberfield, Andrew J.

\section{CITATION:}

Wickham, Shelley F. J.... [et al]. A DNA-based molecular motor that can navigate a network of tracks. Nature Nanotechnology 2012, 7(3): 169173

\section{ISSUE DATE:}

2012-01-22

URL:

http://hdl.handle.net/2433/152384

\section{RIGHT:}

(c) 2012 Macmillan Publishers Limited. All rights reserved.; 許諾条件によ り本文は2012-07-22に公開.; この論文は出版社版でありません。引用 の際には出版社版をご確認ご利用ください。;This is not the published version. Please cite only the published version. 
A DNA-based molecular motor that can navigate a network of tracks.

Shelley F. J. Wickham ${ }^{1}$, Jonathan Bath ${ }^{1}$, Yousuke Katsuda ${ }^{2}$, Masayuki Endo ${ }^{3,4}$, Kumi Hidaka $^{2}$, Hiroshi Sugiyama, ${ }^{2,3,4^{*}}$ and Andrew J. Turberfield ${ }^{{ }^{*}}$

${ }^{1}$ University of Oxford, Department of Physics, Clarendon Laboratory, Parks Road, Oxford OXI 3PU, UK. ${ }^{2}$ Department of Chemistry, Graduate School of Science, Kyoto University, Kitashirakawa-oiwakecho, Sakyo-ku, Kyoto 606-8502, Japan. ${ }^{3}$ Institute for Integrated Cell-Material Sciences (iCeMS), Kyoto University, Yoshidaushinomiyacho, Sakyo-ku, Kyoto 606-8501, Japan. ${ }^{4}$ CREST, Japan Science and Technology Corporation (JST), Sanbancho, Chiyoda-ku, Tokyo 102-0075, Japan. 
Synthetic molecular motors can be fuelled by the hydrolysis ${ }^{1-4}$ or hybridization ${ }^{5-11}$ of DNA. Such motors can move autonomously ${ }^{1-4,7-11}$ and programmably ${ }^{12}$, and longrange transport has been observed on linear tracks ${ }^{13,14}$. It has also been shown that DNA systems can compute ${ }^{8,15-18}$. Here we report a synthetic DNA-based system that integrates long-range transport and information processing. We show that the path of a motor through a network of tracks containing four possible routes can be programmed using instructions that are added externally or carried by the motor itself. When external control is used we find that $87 \%$ of the motors follow the correct path; $71 \%$ of the motors follow the correct path when internal control is used.

Programmable motion will allow the development of computing networks, molecular systems that can sort and process cargos according to instructions that they carry, and assembly lines ${ }^{19,20}$ that can be reconfigured dynamically in response to changing demands.

A network of branching tracks was constructed on a rectangular DNA origami tile ${ }^{21}$. Each tile, $100 \mathrm{~nm} \times 70 \mathrm{~nm}$, is assembled from a single-stranded circular template that is hybridized to and cross-linked by 216 short staple strands to form a raft of 24 parallel DNA double helices. The ends of the staples lie on an approximately hexagonal grid, with $6 \mathrm{~nm}$ spacing, on one face of the tile. The tracks consist of single-stranded DNA anchorages ('stators') extending from the 5' ends of selected staples $^{14}$. The track architecture is shown in Figure 1a and Supplementary Figures 1 and 2. The first section of the track is perpendicular to the origami helices; it then splits into two branches, both at $\sim 60^{\circ}$ to the initial direction (the first layer of control comprising a single node). Two special control stators are positioned directly downstream from the node. Unique address and toehold ${ }^{22}$ sequences allow these control stators to be selectively blocked and unblocked to direct a motor down a particular path (Figure 1c). The branching motif is repeated (the second layer of control comprising two nodes) to give four possible routes through the network (Figure 1a). Motors must take 7 steps to traverse a one-layer track and 12 to traverse a two-layer track.

We use a shorthand $(x, y)$ for a program of motion that involves taking direction $x$ at the first node and $y$ at the second, where $x=\{\mathrm{L}, \mathrm{R}\}$ instructs the motor to turn $\{1 \mathrm{eft}$, right $\}$ at the first node. Stators are labeled according to their position in the programmed sequence of motion: for example, $S_{11}(R, L)$ is the $11^{\text {th }}$ stator along the path that turns right at the first node and left at the second. Addition of instruction strand $i(\mathrm{~L},-)$ unblocks control stator $\mathrm{S}_{5}(\mathrm{~L})$ at the first junction and directs the motor down the left-hand path. Similarly, strand $(\mathrm{R},-)$ unblocks $\mathrm{S}_{5}(\mathrm{R})$ and directs the motor to the right. In two-layer tracks, the junction sequences at the second nodes are repeated: control stators $\mathrm{S}_{11}(\mathrm{~L}, \mathrm{~L})$ and $\mathrm{S}_{11}(\mathrm{R}, \mathrm{L})$ are both unblocked by control strand $\mathrm{i}(-, \mathrm{L})$, and $\mathrm{S}_{11}(\mathrm{~L}, \mathrm{R})$ and $\mathrm{S}_{11}(\mathrm{R}, \mathrm{R})$ are unblocked by $\mathrm{i}(-, \mathrm{R})$. The four final stators are reached by unique paths through the network prescribed by the four possible combinations of instruction strands: [i(L,-)+i(-,L)] leads to destination $\mathrm{S}_{13}(\mathrm{~L}, \mathrm{~L})$; [i(L,) $+\mathrm{i}(-, \mathrm{R})]$ leads to $\mathrm{S}_{13}(\mathrm{~L}, \mathrm{R})$; etc.

The motor ${ }^{3}$ consists of a single strand of DNA that is complementary to a domain common to each stator. The motor-stator duplex contains the recognition site of a nicking restriction enzyme that catalyses hydrolysis of the stator, leading to the dissociation of the cut fragment and exposure of a 6-nucleotide (nt) toehold ${ }^{22}$ that initiates migration of the motor onto an adjacent intact stator ${ }^{5}$ (Figure 1c). The motor is initially hybridized to the first stator $\left(\mathrm{S}_{1}\right)$, which is incorporated in the tile in a final 
assembly step ${ }^{14}$. To assist the loading process, stator $S_{1}$ is designed to hybridize to an additional $2 \mathrm{nt}$ of the motor. All other stators (not just the control stators) are initially occupied by 'block' strands. Block strands prevent binding of the motor to a stator: they hybridize to an identifying address domain at the top of the stator and a short section of the motor-binding domain (10 nt for control stators and $6 \mathrm{nt}$ for all other stators). They also incorporate a single-stranded toehold ${ }^{22}$ that facilitates their removal by addition of a complementary 'unblock' strand with a matching address domain (Figure 1c). All generic stators, but only selected control stators, are unblocked before the motor is activated by addition of enzyme. The blocking strand binding domain does not extend far enough into the enzyme recognition site to allow blocked stators to be cut. Stators at track ends contain a single-base mismatch in the enzyme recognition site that prevents cleavage and thus captures the motor when it reaches the end of the track.

Ensemble fluorescence measurements were used to observe transport of a fluoresence-quenching cargo molecule, attached to the motor, past fluorophores positioned along the track. One-layer tracks were labeled at $\mathrm{S}_{1}, \mathrm{~S}_{8}(\mathrm{~L})$ and $\mathrm{S}_{8}(\mathrm{R})$ with fluorophores $F_{1}, F_{8}(L)$ and $F_{8}(R)$, respectively. Partial tracks containing only one branch were used to confirm that the motor behaves on tracks with $60^{\circ}$ bends as on straight tracks of the same length (Supplementary Figure 3). Figure 2 shows timedependent fluorescence signals from branched tracks. In all cases, $\mathrm{F}_{1}$ intensity increases immediately on addition of enzyme, indicating motion away from $\mathrm{S}_{1}$. Quenching of fluorophores $\mathrm{F}_{8}$ indicates accumulation of the motor at the track ends. When instruction $\mathrm{i}(x)$ is added before transport is initiated, fluorescence from the intended destination $\mathrm{F}_{8}(x)$ is quenched strongly, while fluorescence from the other track-end label is largely unchanged (65\% of motors have reached the end of the track within 200 minutes, of which 76\% reach the correct destination-see Supplementary Information Tables 2 and 3). When both instruction strands are added, $\mathrm{F}_{8}(\mathrm{~L})$ and $\mathrm{F}_{8}(\mathrm{R})$ are quenched equally ( $28 \%$ of motors reach the left end, $28 \%$ the right). These results do not depend on the choice of fluorophores at the track ends (Supplementary Figure 4). This demonstrates that the dominant path taken by the motor is determined by the instructions added, and that when both paths at the junction are open there is no bias between them. Leakage of motor into the 'wrong' path is observed: this is significantly reduced when the control stator for each track branch is repeated at position $\mathrm{S}_{6}$ (of the $56 \%$ of motors that reach the end of the track within 200 minutes, $87 \%$ reach the correct destination, Figure 2). Motors can cross a $12 \mathrm{~nm}$ gap in the track at a much slower rate ${ }^{14}$, and may leak past the block by stepping directly onto the next available downstream stator. Twin-block tracks increase the required stepsize to $18 \mathrm{~nm}$, reducing the leakage rate further ${ }^{14}$. If both downstream paths are blocked, the motor is not trapped indefinitely (Supplementary Figure 5): it either leaks forward through the blocked stators or diffuses back over the cut stators towards $\mathrm{S}_{1}$. Diffusion is slow compared to enzyme-driven stepping ${ }^{14}$, and can be neglected if at least one downstream path is unblocked.

Fluoresence measurements were used to observe motor movement along a two-layer track with twin blocking stators at each node. (The additional control sequence was tested in separate experiments: Supplementary Figure 6). Tiles labeled with $\mathrm{F}_{7}(\mathrm{R})$ at intermediate stator $S_{7}(R)$ on the first right branch, and with $F_{13}(R, L)$ and $F_{13}(R, R)$ at two of the four track ends, were tested with all four instruction sets. The experiment was then repeated with tiles labeled at $F_{7}(R), F_{13}(L, L)$ and $F_{13}(L, R)$. The combined 
results are shown in Figure 3. A dip in $\mathrm{F}_{7}(\mathrm{R})$ fluorescence, indicating passage of the motor along the right branch at the first node, is observed if and only if the instruction for the first node is $\mathrm{i}(\mathrm{R},-)$. The results for the four $\mathrm{F}_{13}$ fluorophores confirm that, in each case, the dominant destination of the motor corresponds to the instructions given ( $67 \%$ of motors that reach the end of the track within 200 minutes have reached the correct destination). The second instruction strand operates on both nodes in the second layer, and directs the motor even if it takes the incorrect path at the first node. For example, addition of program $(R, L)$, results in strong quenching of $F_{13}(R, L)$ as expected ( $65 \%$ of motors reaching the end within 200 minutes are at $\left.S_{13}(R, L)\right)$, but of those motors that take the wrong path at the first node, $69 \%$ are directed correctly to $\mathrm{S}_{13}(\mathrm{~L}, \mathrm{~L})$.

The conclusion that the path chosen by the motor is determined by the control strands is supported by atomic force microscopy (AFM) imaging. Motor strands modified with biotin were labeled with streptavidin after execution of the program, then imaged by AFM to determine the motor location. Representative images are shown in Figure 3 iii. Histograms of final motor positions are consistent with the programs loaded (Figure 3 iv).

In the experiments described above, broadcast instructions are supplied to an ensemble of motors in solution by diffusion. A potentially richer set of behaviors is possible if instructions are carried by the motors themselves. Such a system is outlined in Figure 4a. The block strand was designed with looped secondary structure in order to impede hybridization with the complementary unblock strand: this was found to reduce the uncatalysed reaction rate less significantly than was expected ${ }^{23}$ so a short splice strand was added to the unblock strand to form a two-strand loop complex that further impedes the reaction (Figure 4a, Supplementary Figure 7). The motor incorporates an additional single-stranded DNA domain that is designed to catalyse hybridization between a specific pair of block and unblock strands, opening only one branch of the track. Unblock strands for all control stators are added before motion is initiated but the unblocking reaction is only catalysed when the motor reaches the selected junction (Supplementary Figure 9). Fluorescence measurements show operation of the motor-catalysed system on a single-layer track (Figure 4 and Supplementary Figure 8). A significant bias is observed towards the branch encoded by the catalytic sequence carried by the motor ( $59 \%$ for the instruction $(\mathrm{R}), 70 \%$ for the instruction (L)). A motor can catalyse more than one unblocking reaction: for instruction (R), a twin block causes a small decrease in the fraction of motor reaching the end of the track (from 37\% for a single block to 33\% for a twin block), and a more significant increase in the bias towards the correct destination (from 59\% to 70\%) for motors that do reach the end of the track. For instruction (L), the twin block results in a decrease in the fraction of the motor that reaches the end of the track (from 52\% to $39 \%$ ) but little or no change in the bias towards the correct destination (70\%). The trade off between increasing the fidelity with which instructions are interpreted and decreasing the fraction of motors that traverse the track within a given time must be considered when optimizing this system.

These results demonstrate that a DNA motor can be routed through a spatial network of bifurcating tracks. The path taken at a junction can be externally controlled or programmed by information carried by the motor itself (with $87 \%$ and $71 \%$ of the cargo routed correctly for external and internal control respectively). Track elements 
can be repeated in series to improve efficiency and re-used at different branch points. More complex track networks could be produced by concatenating origami tiles to form larger structures ${ }^{24-27}$. This work represents a significant advance in the creation of programmable and adaptable molecular systems. The capacity to control routing decisions locally, by means of signals carried by the motors that they control, is a particularly significant step: molecular transporters can already operate without the need for external control ${ }^{1-4,7-14}$; they can now process information autonomously, Individual molecular robotic systems that to respond to local stimuli and pass control signals to each other could lead, for example, to the development of responsive systems for distributed drug manufacture and release. These characteristics provide the elements of control required to implement Petri-Net-style computation ${ }^{28}$; they also permit complex collective behaviours such as those that underlie 'social' robotics ${ }^{29}$. 
1. Yin, P., Yan, H., Daniell, X. G., Turberfield, A. J. \& Reif, J. H. A unidirectional DNA walker that moves autonomously along a DNA track. Angew. Chem. Int. Edn 43, 4906-4911 (2004).

2. Tian, Y., He, Y., Peng, Y. \& Mao, C. A DNA enzyme that walks processively and autonomously along a one-dimensional track. Angew. Chem. Int. Edn 44, 4355-4358 (2005).

3. Bath, J., Green, S. J. \& Turberfield, A. J. A free-running DNA motor powered by a nicking enzyme. Angew. Chem. Int. Edn 44, 4358-4361 (2005).

4. Pei, R. et al., Behaviour of polycatalytic assemblies in a substrate-displaying matrix. J. Am. Chem. Soc. 128, 12693-12699 (2006).

5. Shin, J. -S. \& Pierce, N. A. A synthetic DNA walker for molecular transport. J. Am. Chem. Soc. 126, 10834-10835 (2004).

6. Sherman, W. B. \& Seeman, N. C. A precisely controlled DNA biped walking device. Nano Lett. 4, 1203-1207 (2004).

7. S. Venkataraman, S., Dirks, R. M., Rothemund, P. W. K., Winfree, E. \& Pierce, N. A. An autonomous polymerization motor powered by DNA hybridization. Naure Nanotech. 2, 490-494 (2007).

8. Yin, P., Choi, H. M., Calvert, C. R. \& Pierce, N. A. Programming biomolecular self-assembly pathways. Nature 451, 318-322 (2008).

9. Green, S. J., Bath, J. \& Turberfield, A. J. Coordinated chemomechanical cycles: a mechanism for autonomous molecular motion. Phys. Rev. Lett. 101, 238101 (2008).

10. Bath, J. Green, S. J., Allen, K. E. \& Turberfield, A. J. Mechanism for a directional, processive and reversible DNA motor. Small 5, 1513-1516 (2009).

11. Omabegho, T., Sha, R. \& Seeman, N. C. A bipedal Brownian motor with coordinated legs. Science 324, 67-71 (2009).

12. Muscat, R. A., Bath, J. \& Turberfield, A. J. A Programmable Molecular Robot. Nano Lett. 11, 982-987 (2011).

13. Lund, K. et al., Molecular robots guided by prescriptive landscapes. Nature 465, 206-210 (2010).

14. Wickham, S. F. J., Endo, M., Katsuda, Y., Hidaka, K., Bath, J., Sugiyama, H. \& Turberfield, A. J. Direct observation of stepwise movement of a synthetic molecular transporter. Nature Nanotech. 6, 166-169 (2011).

15. Stojanovic, M. N., Mitchell, T. E. and Stefanovic, D. Deoxyribozyme-based logic gates. J. Am. Chem. Soc. 124, 3555-3561 (2002).

16. Zhang, D. Y., Turberfield, A. J., Yurke, B. \& Winfree, E. Engineering entropydriven reactions and networks catalyzed by DNA. Science 318, 1121-1125 (2007). 
17. Benenson, Y., Gil, B., Adar, U. B.-D. R. and Shapiro, E. An autonomous molecular computer for logical control of gene expression. Nature 429, 423-429 (2004).

18. Qian, L. \& Winfree, E. Scaling Up Digital Circuit Computation with DNA Strand Displacement Cascades. Science 332, 1196-1201 (2011).

19. He, Y. and Liu, D. R. Autonomous multistep organic synthesis in a single isothermal solution mediated by a DNA walker. Nature Nanotech. 5, 778-782 (2010).

20. Gu, H., Chao, J., Xiao, S. J. \& Seeman, N. C. A proximity-based programmable DNA nanoscale assembly line. Nature 465, 202-205 (2010).

21. Rothemund, P. W. K. Folding DNA to create nanoscale shapes and patterns. Nature 440, 297-302 (2006).

22. Yurke, B. \& Mills Jr, A. P. Using DNA to power nanostructures. Genetic Programming and Evolvable Machines 4, 111-122 (2003).

23. Turberfield, A. J. et al. DNA fuel for free-running nanomachines. Phys. Rev. Lett. 90, 118102 (2003).

24. Douglas, S. M. et al., Self-assembly of DNA into nanoscale three-dimensional shapes. Nature 459, 414-418 (2009).

25. Endo, M., Sugita, T., Katsuda, Y., Hidaka, K. \& Sugiyama, H. ProgrammedAssembly System Using DNA Jigsaw Pieces. Chem. Eur. J. 16, 5362-5368 (2010).

26. Rajendran, A., Endo, M., Katsuda, Y., Hidaka, K. \& Sugiyama H. Programmed Two-Dimensional Self-Assembly of Multiple DNA Origami Jigsaw Pieces. $A C S$ Nano 5, 665-671 (2011).

27. Liu, W., Zhong, H., Wang, R. \& Seeman, N. C. Crystalline Two-Dimensional DNA-Origami Arrays. Angew. Chem. Int. Edn 50, 264-267 (2011).

28. Petri, C. A. Kommunikation mit Automaten. Bonn: Institut fur Instrumentelle Mathematik, Schriften des IIM Nr. 3 (1962).

29. Krieger, M. J. B., Billeter, J. B. \& Keller, L. Ant-like task allocation and recruitment in cooperative robots. Nature 406, $992-995$ (2000). 
Acknowledgements. This work was supported by the Engineering and Physical Sciences Research Council (EP/G037930/1), the Clarendon Fund, the OxfordAustralia Scholarship Fund, the CREST of JST and a Grant-in-Aid for Science Research from the Ministry of Education, Culture, Sports, Science and Technology, Japan.

Author contributions. Experiments were designed by S.W. with input from J.B. and A.J.T. Ensemble fluorescence experiments were carried out by S.W. in the laboratory of A.J.T.. AFM experiments were done by M.E., Y.K. and K.H in the laboratory of H.S. The manuscript was written by S.W., J.B., H.S. and A.J.T.

Additional information. The authors declare no competing financial interests. Supplementary information accompanies this paper at www.nature.com/naturenanotechnology. Reprints and permission information is available online at http://npg.nature.com/reprintsandpermissions/. Correspondence and requests for materials should be addressed to H.S. and A.J.T. 
Figure 1. Programmed route on branching tracks. a The DNA track network is assembled on a rectangular DNA origami substrate. Selective displacement of blocking strands from junction stators (coloured crosses) opens just one path. The motor (black circle) travels down the open path, destroying the track behind it. b Tracks decorated with excess motor visualized by AFM (scale bars $50 \mathrm{~nm}$ ). A reference marker (black square) is used to confirm the orientation of the track $\mathbf{c}$ 'Block' strands with unique address domains (magenta/green) prevent the motor (black) from stepping when it reaches a junction. The selected path is unblocked by an instruction strand which hybridizes to the toehold on the selected block strand (green) to initiate a strand displacement reaction that removes it from the stator. The motor can then step to the unblocked stator. The resulting duplex contains a new recognition site for the nicking enzyme: enzyme cleavage of the stator, and subsequent dissociation of the cut stator fragment, generates a 6-nt toehold that initiates migration of the motor onto the next intact stator. Repetition of this cycle of step and cut drives the motor along the programmed path. 
Figure 2: Externally controlled DNA motor on a single-layer track. The motor transports a fluorescence quencher past fluorophores $\mathrm{F}_{1}, \mathrm{~F}_{8}(\mathrm{R})$ and $\mathrm{F}_{8}(\mathrm{~L})$, labeling stators at the beginning and ends of the track. a Addition of instruction $\mathrm{i}(\mathrm{R})$,

unblocking the control stator on the right-hand branch, directs the motor to $S_{8}(R)$ and results in strong quenching of $\mathrm{F}_{8}(\mathrm{R})$. b i $(\mathrm{L})$ directs the motor to $\mathrm{S}_{8}(\mathrm{~L})$, where it quenches $F_{8}(L)$. c If both paths are open the motor quenches $F_{8}(R)$ and $F_{8}(L)$ equally. Leakage into the wrong branch is decreased for 'twin-block' tracks, where each control stator is repeated at successive positions downstream from the node. 
Figure 3. Controlled motion on a two-layer track. Four instruction strands are used to control the path taken by the motor, which transports a fluorescence quencher past fluorophores positioned along the track. a Time-dependent fluoresence intensities (i) and diagram showing programmed route (ii) on addition of instruction set $(\mathrm{R}, \mathrm{R})$. The right hand path at both junctions is opened, and the motor is directed to stator $\mathrm{S}_{13}(\mathrm{R}, \mathrm{R})$ where it quenches fluorophore $\mathrm{F}_{13}(\mathrm{R}, \mathrm{R})$. iii AFM image of a streptavidinlabeled motor $(\mathrm{M})$ showing its final location at the programmed track end, $\square$ indicates a reference marker. A height profile of this image is shown in Supplementary Figure 10. iv Histogram showing proportions of motors detected by AFM at each track end (number of tiles counted, $\mathrm{N}=21$ ). Similar results indicate correctly programmed motion for the other instruction sets: $\mathbf{b}(\mathrm{R}, \mathrm{L}), \mathrm{N}=22$; $\mathbf{c}(\mathrm{L}, \mathrm{R}), \mathrm{N}=21$ and $\mathbf{d}(\mathrm{L}, \mathrm{L})$, $\mathrm{N}=25$. A transient dip in $\mathrm{F}_{7}(\mathrm{R})$ is seen only if the instruction set contains $(\mathrm{R},-)$. (Scale bars $20 \mathrm{~nm}$.) 
Figure 4. Internally programmed motion on a single-layer track. a The motor carries a catalytic domain that can only unblock a path with complementary address domains (magenta). A $6 \mathrm{nt}$ toehold carried by the motor (M) initiates a strand exchange reaction that partially displaces the blocking strand (B) from the adjacent stator revealing a domain that was previously sequestered in a loop. The open loop interacts with the unblock•splice $(\mathrm{U} \bullet \mathrm{S})$ complex to nucleate a four-arm Holliday junction. Resolution of the junction removes the unblocking strand from the stator leaving the shorter splice domain in its place. Note that the nicking enzyme places some restriction on the design of the blocking and splice strands: the block•stator duplex must not contain a recognition sequence and the splice•stator duplex must reveal a toehold to allow the motor to step. Transfer of the motor to the unblocked stator proceeds as described in Fig. 1 except that 2 nt of the $6 \mathrm{nt}$ toehold remain obscured by the splice domain. $\mathbf{c}$ Motor(R) carries the instruction to open the righthand path, and is directed to $S_{8}(R)$, quenching $F_{8}(R)$. d Motor $(L)$ quenches $F_{8}(L)$. e A motor carrying no catalytic instruction domain is split between the two paths. Leakage into the wrong state is decreased for twin-block tracks, which have two independently blocked stators on each path downstream from the node. 
a

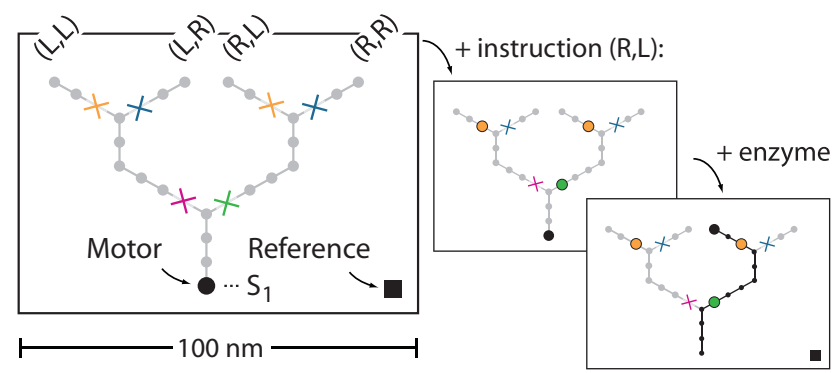

b
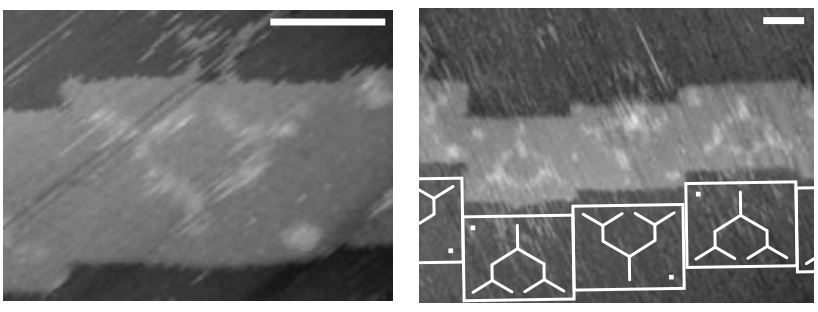

c

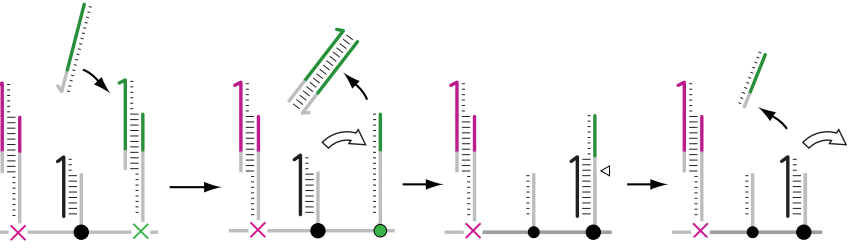




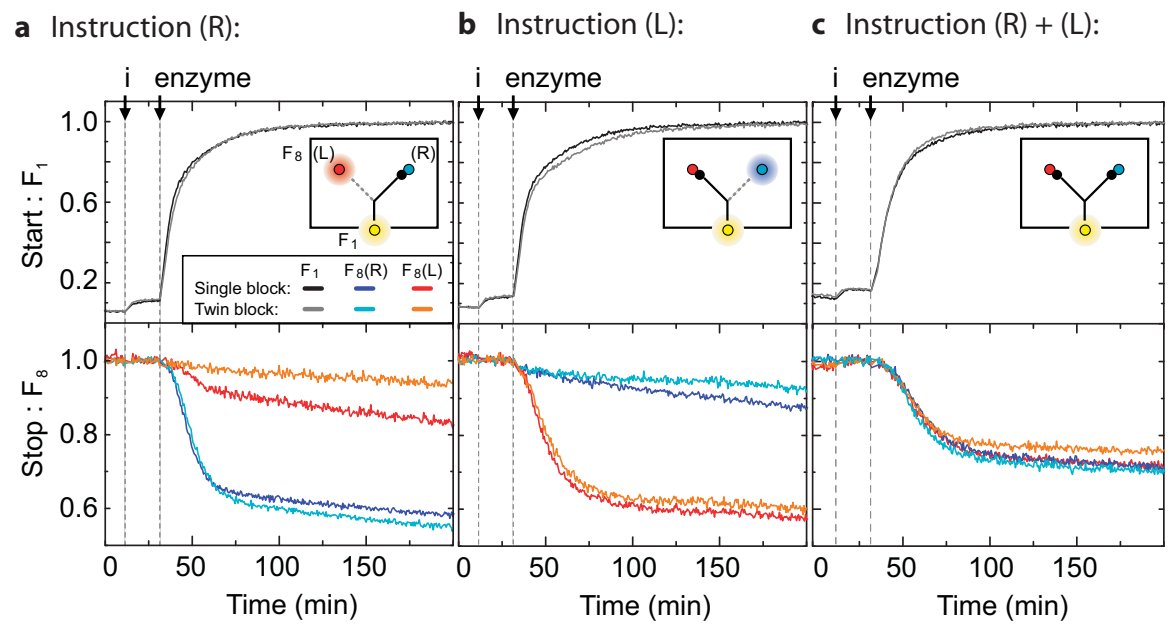


a Instruction $(R, R)$ :

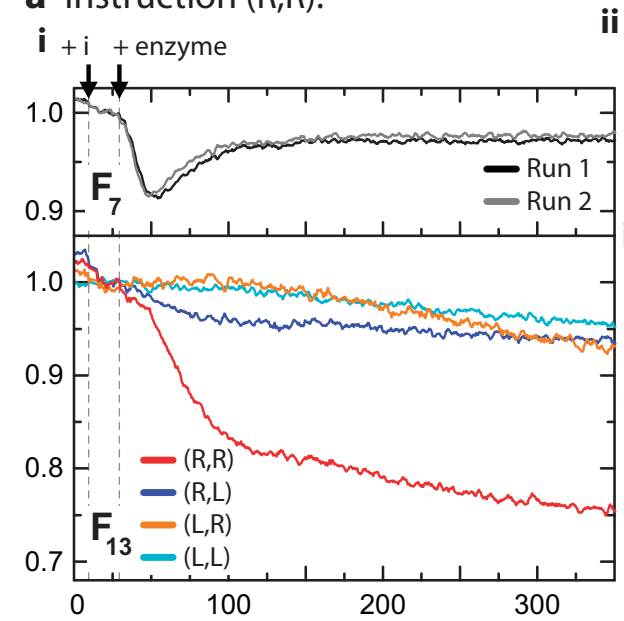

c Instruction (L,R):

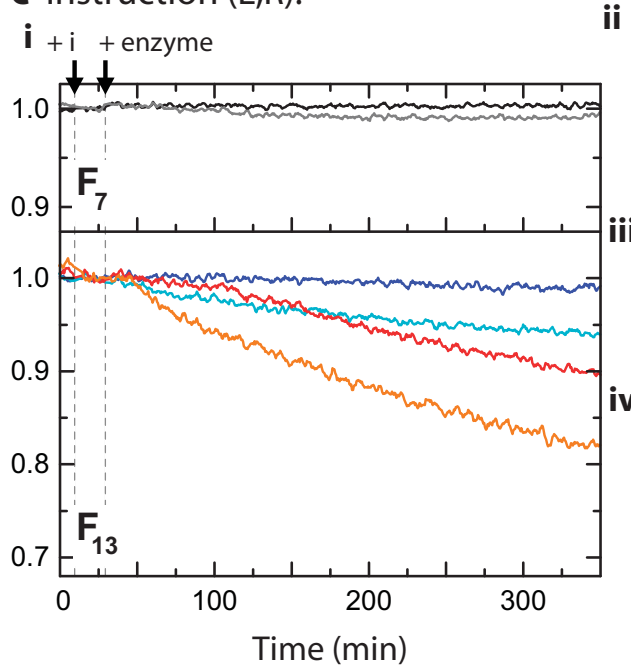

b Instruction $(\mathrm{R}, \mathrm{L})$ :

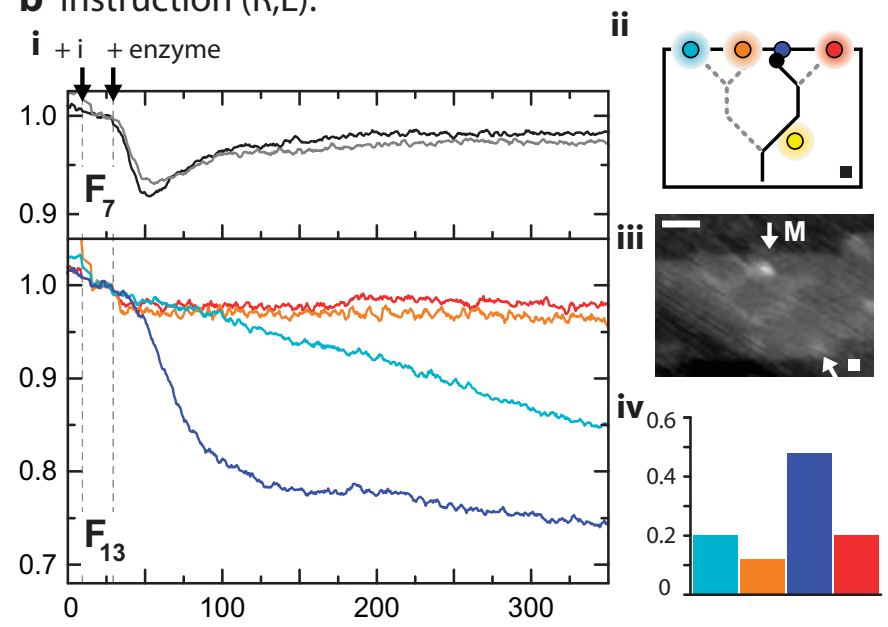

d Instruction (L,L):

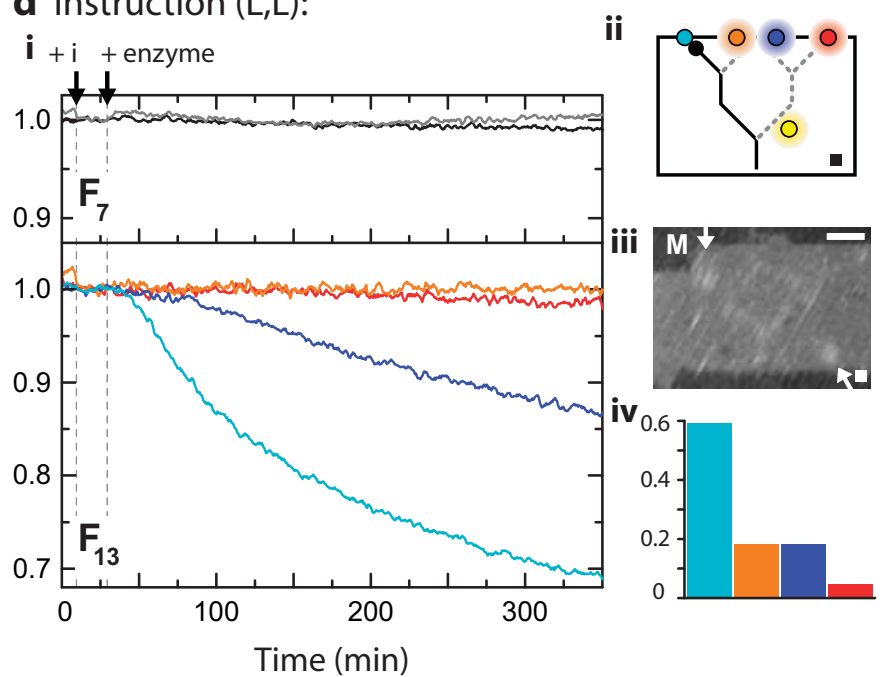


a
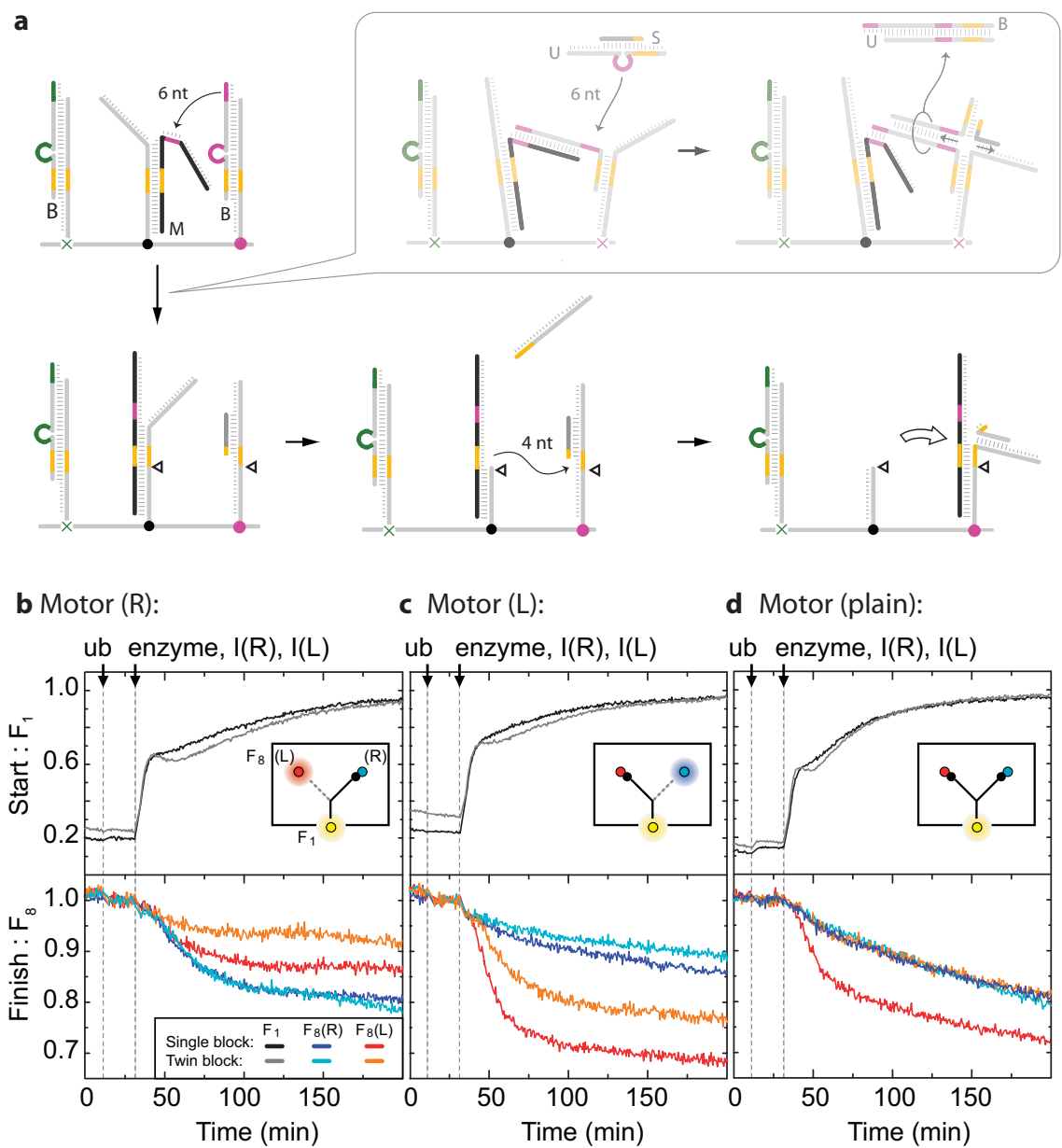


\section{A DNA-based molecular motor that can navigate a network of tracks (Supplementary Information).}

Shelley F. J. Wickham, Jonathan Bath, Yousuke Katsuda, Masayuki Endo, Kumi Hidaka, Hiroshi Sugiyama, and Andrew J. Turberfield

1. Supplementary Methods

2. Supplementary Figures

Supplementary Figure 1: Single-layer track layout.

Supplementary Figure 2: Two-layer track layout.

Supplementary Figure 3: 1-D tracks with a corner.

Supplementary Figure 4: Single-layer track with transposed fluorophores.

Supplementary Figure 5: Single-layer track with both paths blocked.

Supplementary Figure 6: Additional control domains for the two-layer track.

Supplementary Figure 7: Design of the motor-catalysed unblocking system.

Supplementary Figure 8: Motor-catalysed system: additional fluorescence results.

Supplementary Figure 9: Internal instructions act locally.

Supplementary Figure 10: Height profiles for AFM data in Figure 3.

3. Supplementary Tables

Supplementary Table 1: DNA sequences for modified staples, motor and track.

Supplementary Table 2: Bias observed for single-layer tracks.

Supplementary Table 3: Bias observed for two-layer tracks 


\section{Supplementary Methods}

DNA Origami rectangle. The DNA origami rectangle was based on the published design for a rectangle with central seam [21], modified to reduce global twist [14]. The tile consists of 72 32-nt staples and 144 31-nt staples, as listed in [14]. The tile has 24 282-bp helices cross-linked on average every $15.6 \mathrm{bp}$, corresponding to 10.4 bp/turn.

Origami synthesis. Single-stranded M13mp18 DNA was ordered from USB (Affymetrix) as the template strand for the origami rectangle. Staple strands were purchased from Integrated DNA Technologies (IDT). Unmodified staples were purchased unpurified. Stator-modified staples were purchased PAGE-purified. Fluorescently labeled strands (JOE, Cy5, FAM) and motor (IowaBlackRQ) were purchased HPLC-purified. Strands fluorescently labeled with Cy3.5 or Alexa594 were purchased unpurified with $3^{\prime}$ amine modification and conjugated to Cy3.5 NHS ester dye (GE Healthcare), or Alexa FLUOR 594 (Invitrogen) following manufacturers instructions, then purified by reverse-phase HPLC (Waters). The solution for origami synthesis contained $50 \mathrm{nM} \mathrm{M13mp18}$ and $250 \mathrm{nM}$ of each staple strand except $\mathrm{S}_{1}$ (5fold excess) in tris-acetate buffer with $12.5 \mathrm{mM}$ magnesium acetate $(\mathrm{pH} 8.3)$. The resulting solution was annealed from $95^{\circ} \mathrm{C}$ to $20^{\circ} \mathrm{C}$ in a PCR machine (Eppendorf) at an average rate of $1^{\circ} \mathrm{C} /$ minute.

Motor Loading. The $S_{1}+$ motor duplex was pre-annealed by combining the stator $S_{1}$ and motor at ratio $1: 0.95$, heating to $95^{\circ} \mathrm{C}$, and cooling to $20^{\circ} \mathrm{C}$ in a PCR machine at a rate of $7.5^{\circ} \mathrm{C} /$ minute. Origami tiles were annealed without the staple corresponding to stator $\mathrm{S}_{1}$, then incubated with a sub-stoichiometric quantity $(0.9 \times)$ of the $\mathrm{S}_{1}+$ motor duplex for 1 hour at $37^{\circ} \mathrm{C}$ to load the motor at the start of the track.

Origami purification. Excess staples were separated from the origami by gel filtration through hand-packed columns using Sephacryl S-300 HR (GE Healthcare). This is a size-exclusion resin with DNA exclusion limit of $118 \mathrm{bp}$. Origami samples of up to $60 \mu \mathrm{L}$ were purified 3 times, on columns of $\sim 500 \mu \mathrm{L}$ resin volume, by 
centrifugation at $1000 \mathrm{~g}$ for 4 minutes. This gave a low background level for fluorescence measurements with only a small loss in sample.

Enzyme. Nicking enzyme Nt.BbvCI was purchased from New England Biolabs and added to the purified origami samples, such that the final solution contained 10 units of enzyme, in tris-acetate buffer with $12.5 \mathrm{mM}$ magnesium acetate and $50 \mathrm{mM} \mathrm{NaCl}$ (pH 8.3), in a final volume of $140 \mu \mathrm{L}$.

Fluorescence measurements. Time-dependent fluorescence from origami track samples was measured in a Cary Eclipse Fluorescence Spectrometer (Varian) in $100 \mu \mathrm{L}$ quartz cuvettes (Starna). Mineral oil (Sigma) was added to cuvettes to prevent evaporation. Excitation and emission wavelengths were as follows: Cy3 (545 nm/565 $\mathrm{nm}), \operatorname{Cy5}(645 \mathrm{~nm} / 665 \mathrm{~nm})$, Cy3.5 (575 nm/595 nm), JOE (529 nm/555 nm), Alexa594 (590 nm/618 nm). Slit widths were $5 \mathrm{~nm}$ (excitation) and $10 \mathrm{~nm}$ (emission). Fluorescence intensities were measured every $10 \mathrm{~s}$, with an integration time of $1 \mathrm{~s}$. Sample concentrations used were in the range $15 \mathrm{nM}-25 \mathrm{nM}$. Initial fluorescence signals $(100 \%)$ and the signals in the presence of excess motor $(0 \%)$ were used to set the base line and to normalize signals from different fluorophores. Blocked tracks were activated, by adding approximately $200 \times$ excess of the unblock strand, 20 minutes before initiation of active motor transport by addition of the nicking enzyme. Motor-catalysed unblock strands were added in $\sim 50 \times$ excess at the same time as enzyme activation. All experiments were run at $37^{\circ} \mathrm{C}$. For the results presented in Figs 2 and 4, $\mathrm{F}_{1}$ was labeled with $\mathrm{JOE}, \mathrm{F}_{8}(\mathrm{~L})$ with $\mathrm{Cy} 5$ and $\mathrm{F}_{8}(\mathrm{R})$ with $\mathrm{Cy} 3.5$. For the results presented in Fig. 3, data from tiles labeled with $\mathrm{JOE}$ at $\mathrm{F}_{7}(\mathrm{R}), \mathrm{Cy} 5$ at $\mathrm{F}_{13}(\mathrm{R}, \mathrm{L})$ and Alexa594 at $\mathrm{F}_{13}(\mathrm{R}, \mathrm{R})$ were combined with data from tiles labeled with JOE at $\mathrm{F}_{7}(\mathrm{R}), \mathrm{Cy} 5$ at $\mathrm{F}_{13}(\mathrm{~L}, \mathrm{~L})$ and Alexa594 at $\mathrm{F}_{13}(\mathrm{~L}, \mathrm{R})$.

Optimization of motor-catalysed unblock system. This system (Supplementary Figure 7) required careful tuning of reaction rates. The interaction of the block and unblock must be slow in the absence of motor, to prevent the control stators at the junction from being opened before the arrival of the motor. It must also be fast enough in the presense of the motor to open a path before the motor can leak through 
the blocked stators or diffuse away from the node. Different loop $(6,8,10 \mathrm{nt})$ and neck lengths $(5,7,8 \mathrm{nt})$ were used to tune reaction rates. Fluoresence results for selected loop and neck lengths are shown for the single-layer track (Supplementary Figure 8). For unblock strands without a splice linker (Supplementary Figure 7), very little directional bias was observed in fluorescence measurements (Supplementary Figure 8 i). Unblock strands hybridized to splice strands, with $8 \mathrm{nt}$ for one neck domain and either 5 or $7 \mathrm{nt}$ for the second domain, were assessed (Supplementary Figure $7 \mathrm{~B}$ ). Use of a splice strand significantly increased the directional bias observed (Supplementary Figure 8 ii-iii). Two orthogonal sets of block, unblock and catalyst sequences were designed, one each for the left and right control stators downstream of the junction. The length of the second splice neck domain was tuned to give the best results for each side of the junction. The results in Figure 4 are for a 6-nt loop, with $\mathrm{i}(\mathrm{R})+(8+7)$-nt splice and $\mathrm{i}(\mathrm{L})+(8+5)$-nt splice.

AFM sample preparation. A sample solution ( $40 \mu \mathrm{L})$ containing $10 \mathrm{nM}$ M13mp18 single-stranded template (New England Biolabs), $50 \mathrm{nM}$ staple DNA strands (omitting $\mathrm{S}_{1}$ ), $20 \mathrm{mM}$ Tris- $\mathrm{HCl}$ (pH 7.6), $1 \mathrm{mM}$ EDTA, and $10 \mathrm{mM} \mathrm{MgCl}_{2}$ was annealed from $85^{\circ} \mathrm{C}$ to $15^{\circ} \mathrm{C}$ at a rate of $1.0^{\circ} \mathrm{C} / \mathrm{min}$. The $\mathrm{S}_{1}$-motor duplex and the control-stator-block duplexes were added $(10 \mathrm{nM})$ and the sample was annealed from $30^{\circ} \mathrm{C}$ to $15^{\circ} \mathrm{C}$ at $1.0^{\circ} \mathrm{C} / \mathrm{min}$. To remove selected stator blocks, the corresponding unblock strands $(100 \mathrm{nM})$ were incubated with the DNA tiles at $30^{\circ} \mathrm{C}$ for $1 \mathrm{hr}$. Samples were then purified as described above. Nicking enzyme Nt.BbvCI (20 units) (New England Biolabs) in a $20 \mu \mathrm{L}$ solution containing $20 \mathrm{mM}$ Tris- $\mathrm{HCl}$ (pH 7.6), 1 $\mathrm{mM}$ EDTA and $10 \mathrm{mM} \mathrm{MgCl}$ was added and incubated at $23^{\circ} \mathrm{C}$ for 5 hours.

AFM experiments. AFM images were obtained using a high-speed AFM imaging system (Nano Live Vision, RIBM, Tsukuba, Japan) with a silicon nitride cantilever (Olympus BL-AC10EGS). Samples $(2 \mu \mathrm{L})$ were adsorbed onto a freshly cleaved mica surface for $5 \mathrm{~min}$ at room temperature, incubated with streptavidin (Sigma) for 10 minutes, and then washed three times using the buffer solution used for sample preparation. Scanning was performed in tapping mode at $23^{\circ} \mathrm{C}$ in the sample preparation buffer. Only origami tiles with a streptavidin-labeled motor-strand at the 
end of one of the four tracks were counted. Images with no visibly labeled motor or with insufficient resolution were discarded. Tiles with motor at any point other than the far end of the track were also discarded. For the four instruction strand pairs, $(\mathrm{R}, \mathrm{R}),(\mathrm{R}, \mathrm{L}),(\mathrm{L}, \mathrm{R}),(\mathrm{L}, \mathrm{L}), \mathrm{N}=21,22,21,25$ tiles were counted. 


\section{Supplementary Figures}

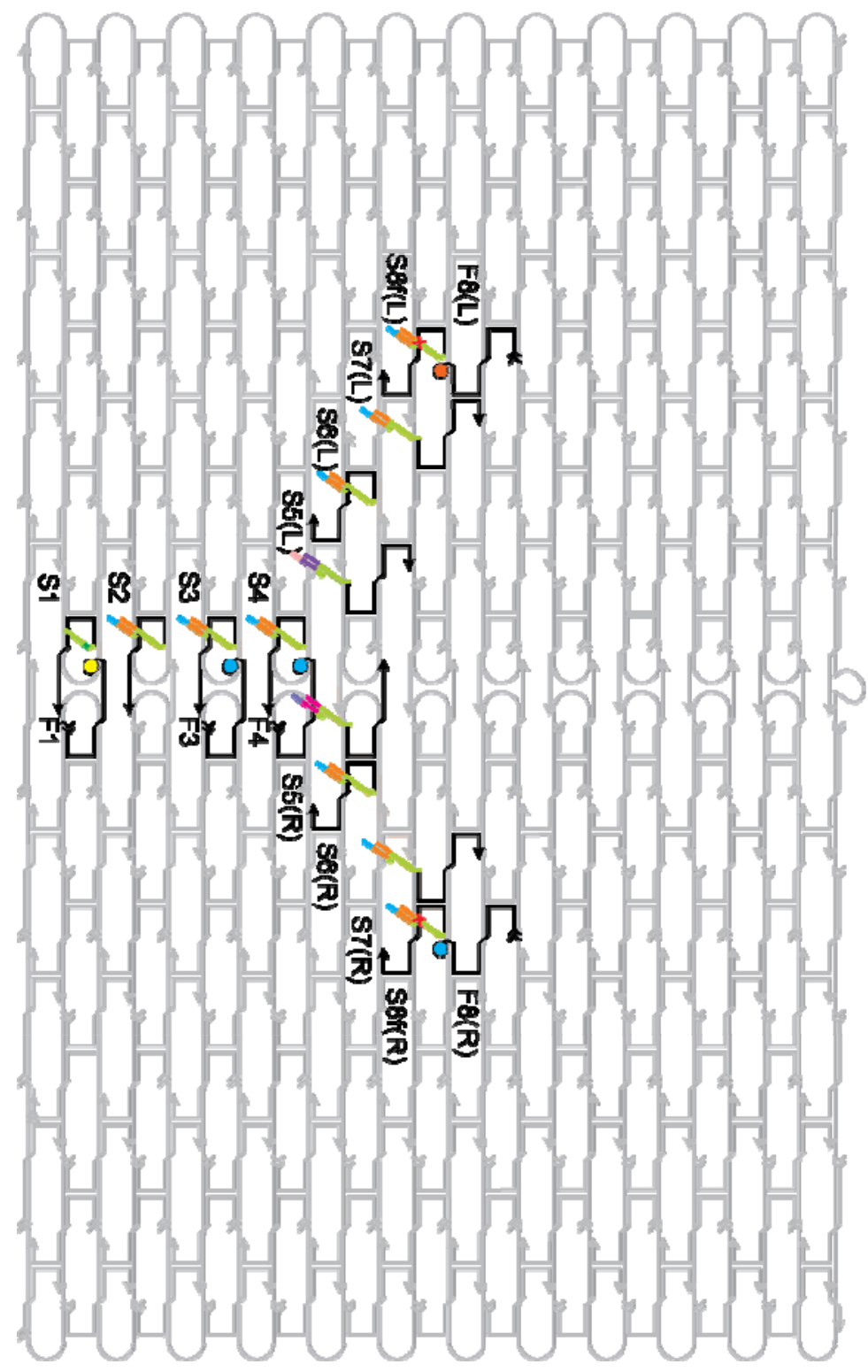

Supplementary Figure 1: Single-layer track layout. Modified origami staples are labeled. The two independently blocked control stators, $\mathrm{S}_{5}(\mathrm{R})$ and $\mathrm{S}_{5}(\mathrm{~L})$, occur directly after the node stator $S_{4}$. The fluorophore-labeled staples $F_{1}, F_{3}, F_{4}, F_{8}(L)$ and $\mathrm{F}_{8}(\mathrm{R})$ are used to observe transport of a quenching molecule along the track. 


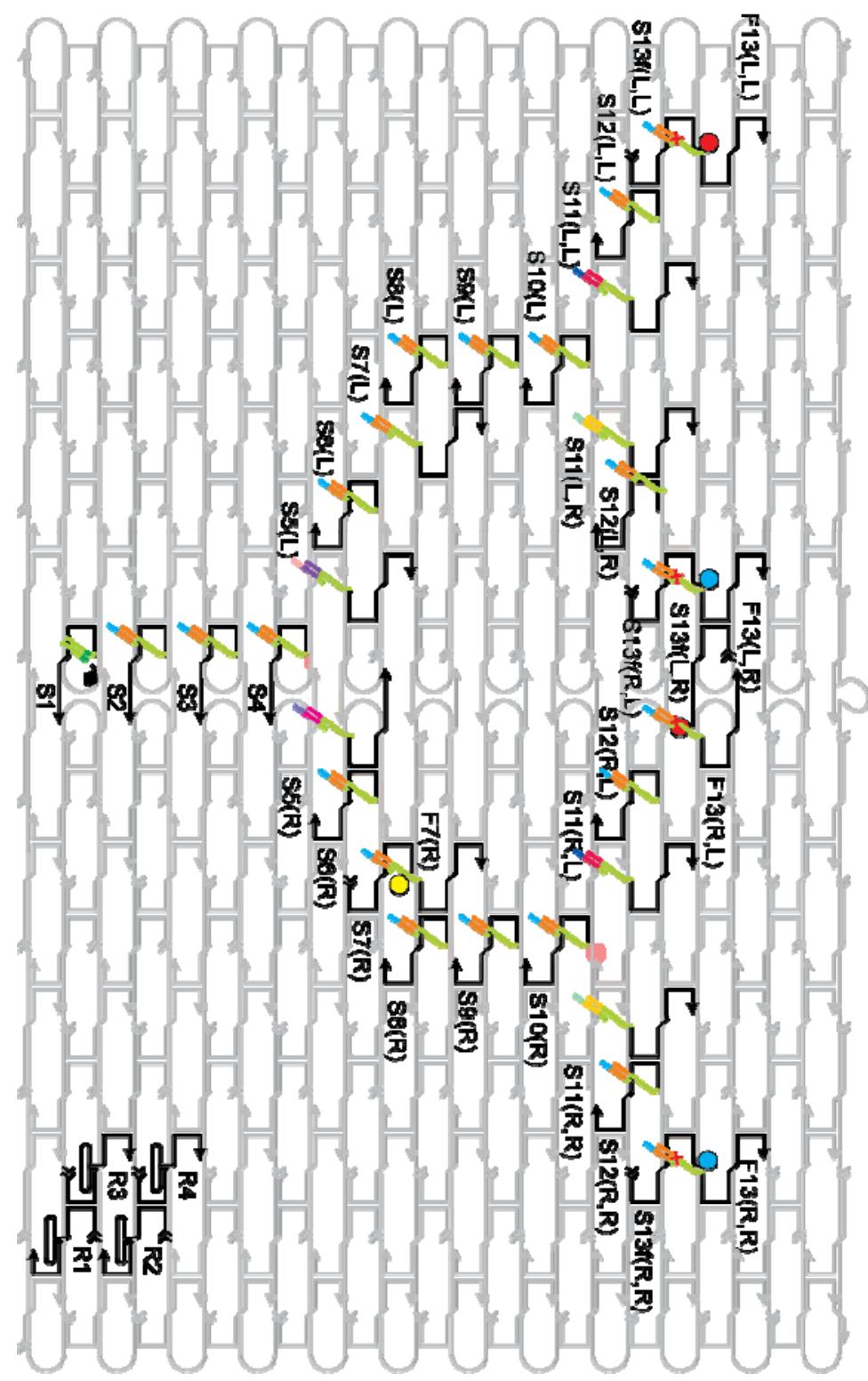

Supplementary Figure 2: Two-layer track layout. Modified origami staples are labeled. Pairs of independently blocked control stators occur directly after each node stator. The same set of sequences (binding, block and unblock) is used at both junctions in the second layer, i.e. after $S_{10}(R)$ and $S_{10}(L)$. Fluorophores $F_{13}(R, R)$, $\mathrm{F}_{13}(\mathrm{R}, \mathrm{L}), \mathrm{F}_{13}(\mathrm{~L}, \mathrm{R})$ and $\mathrm{F}_{13}(\mathrm{~L}, \mathrm{~L})$ label the ends of the 4 possible motor paths. Fluorophore $F_{7}(R)$ indicates the path taken by the motor at the first node. Hairpin modified staple strands $\left(\mathrm{R}_{1}, \mathrm{R}_{2}, \mathrm{R}_{3}, \mathrm{R}_{4}\right)$ are used as reference markers in AFM measurements. 

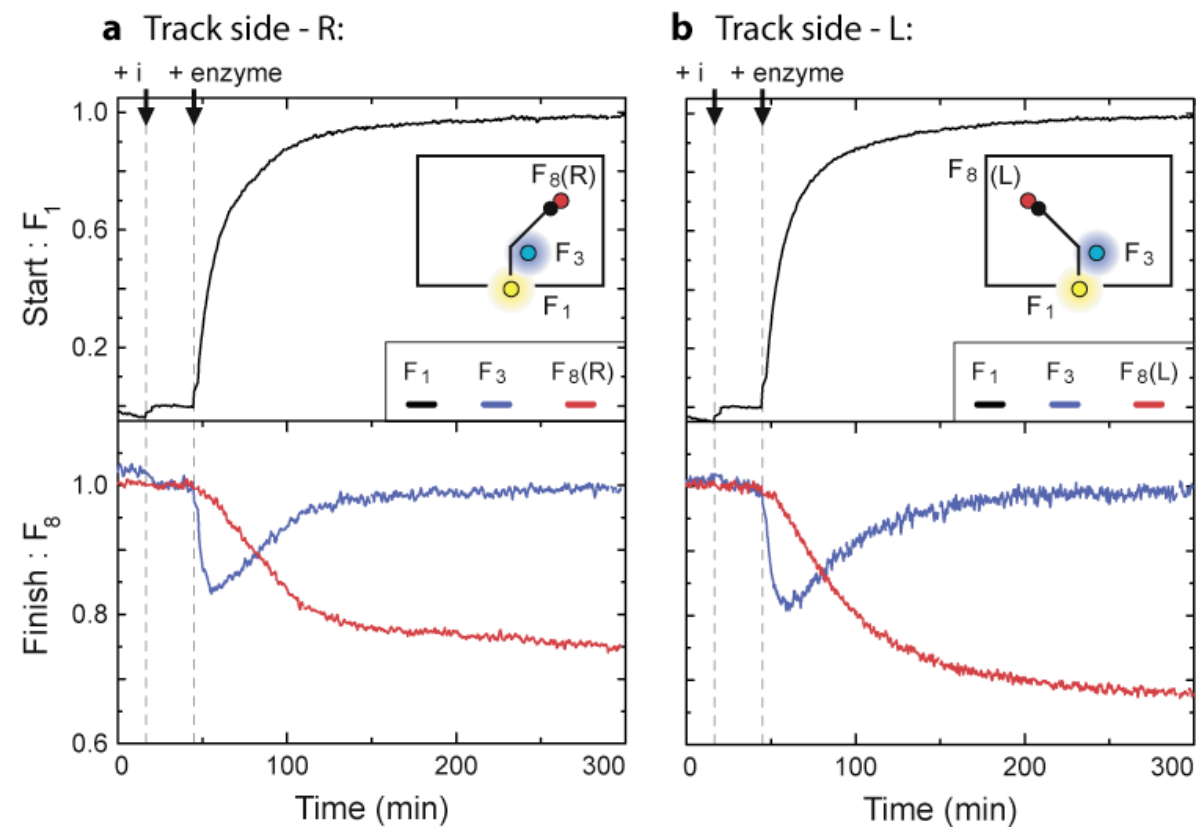

Supplementary Figure 3: 1-D tracks with a corner. The two halves of the single-layer track were tested separately. a The Right half-track tile, $\mathrm{S}_{1}-\mathrm{S}_{8}(\mathrm{R})$. b The Left halftrack tile, $\mathrm{S}_{1}-\mathrm{S}_{8}(\mathrm{~L})$. Time-dependent fluorescence intensities for fluorophores $\mathrm{F}_{1}, \mathrm{~F}_{3}$ and $\mathrm{F}_{8}(\mathrm{R})$ or $\mathrm{F}_{8}(\mathrm{~L})$ are shown. Fluorescence results for these 'bent' tracks are similar to those for a straight track of the same length [14]. $F_{1}$ increases sharply on addition of enzyme as the motor moves away from $S_{1}$. There is a transient dip in $F_{3}$ as the motor moves past $\mathrm{S}_{3}$, and then permanent quenching of $\mathrm{F}_{8}$ as the motor accumulates at the final mis-match stator, $\mathrm{S}_{8}$. Both tracks were unblocked by the addition of the generic unblock strand, and either i(R) or i(L), 20 min before addition of enzyme. 

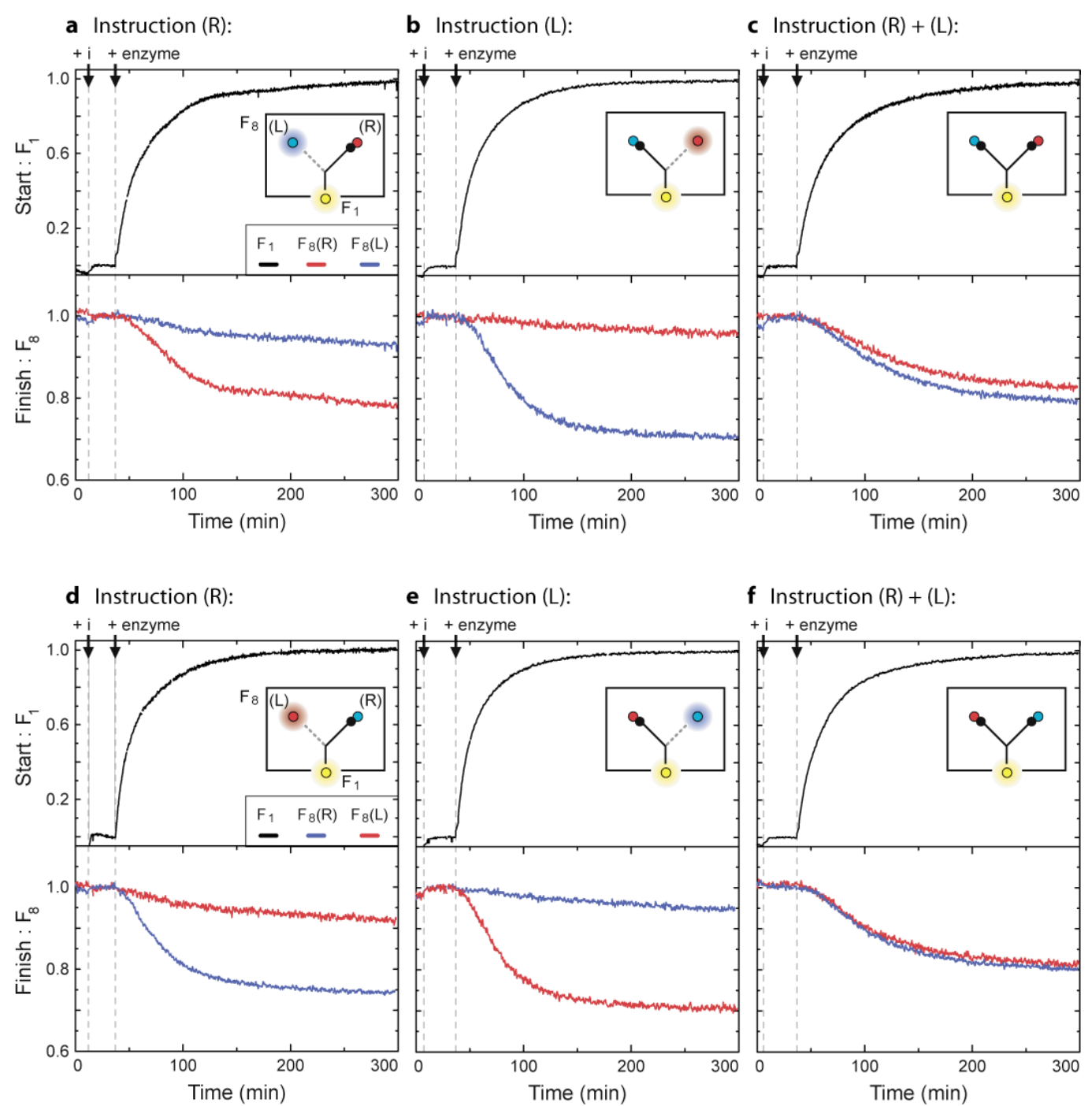

Supplementary Figure 4: Single-layer track with transposed fluorophores. a-c Motor operation observed using fluorophores $F_{1}(\mathrm{Joe}), \mathrm{F}_{8}(\mathrm{~L})(\mathrm{Cy} 3.5)$ and $\mathrm{F}_{8}(\mathrm{R})(\mathrm{Cy} 5)$. d-f Equivalent results with fluorophores $\mathrm{F}_{1}(\mathrm{Joe}), \mathrm{F}_{8}(\mathrm{~L})(\mathrm{Cy} 5)$ and $\mathrm{F}_{8}(\mathrm{R})(\mathrm{Cy} 3.5)$. The observed behaviour of the motor is not changed by transposing fluorophores $F_{8}(L)$ and $F_{8}(R): F_{8}(L)$ is significantly quenched only if $i(L)$ is added, and $F_{8}(R)$ only if $i(R)$ is added. If both $i(L)$ and $i(R)$ are added, $F_{8}(L)$ and $F_{8}(R)$ are quenched equally. 

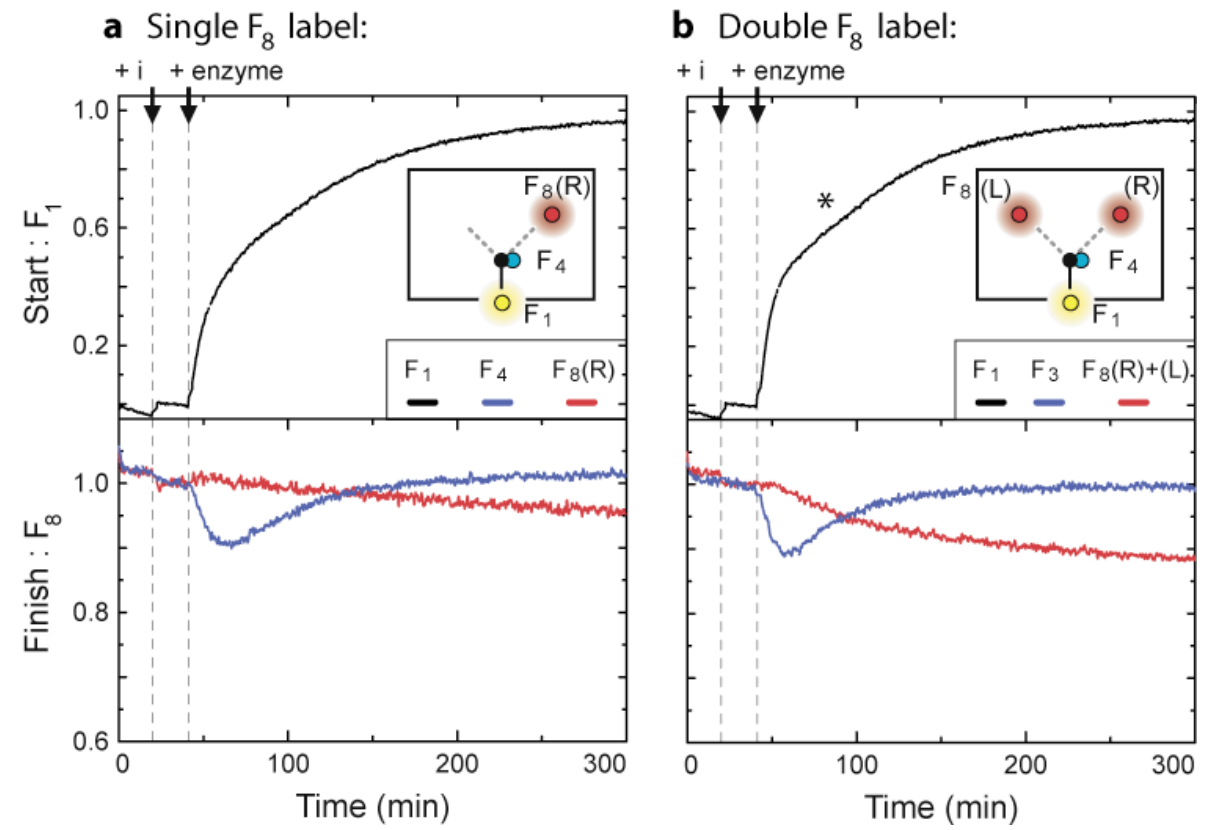

Supplementary Figure 5: Single-layer track with both paths blocked. Tiles are labeled with $F_{1}$ and $F_{4}$ and either a $F_{8}(R)$, or b both $F_{8}(R)$ and $F_{8}(L)$. Quenching of $F_{4}$ indicates arrival of the motor at the node stator $\mathrm{S}_{4}$. However, the motor is not indefinitely trapped at this position by the blocked downstream stators, as the signal slowly recovers. Quenching of $\mathrm{F}_{8}(\mathrm{R})$ and $\mathrm{F}_{8}(\mathrm{~L})$, indicates that some motor leaks forward into the downstream paths. The slight dip in the $F_{1}$ curve (marked*) is consistent with the diffusion of blocked motor upstream, back to $S_{1}$, which binds to the motor by an additional $2 \mathrm{nt}$ compared to other cut stators. 
a Instruction $(-, \mathrm{R})$ :
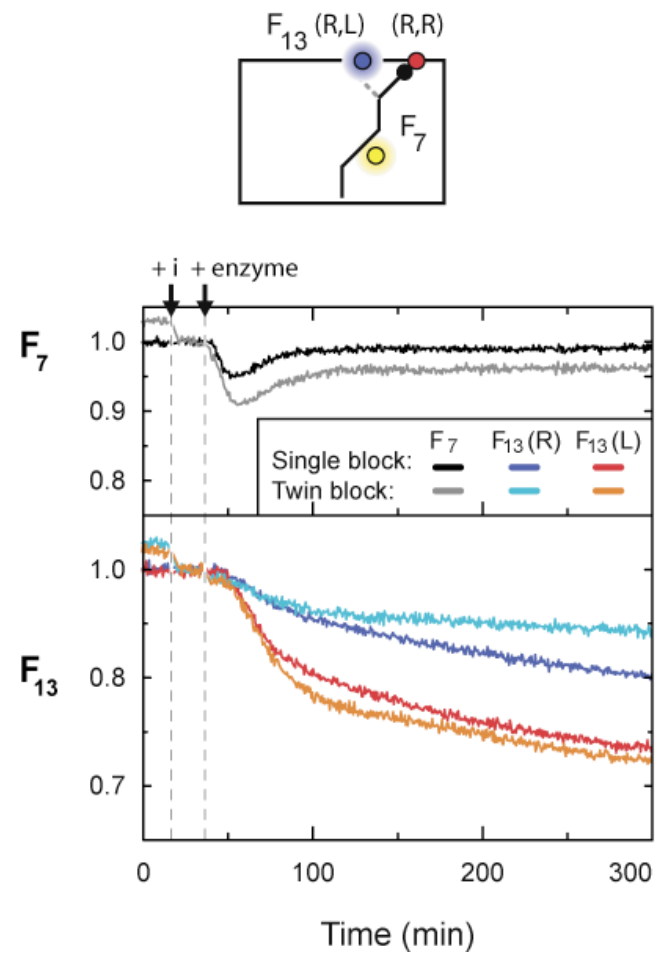

b Instruction $(-, \mathrm{L})$ :
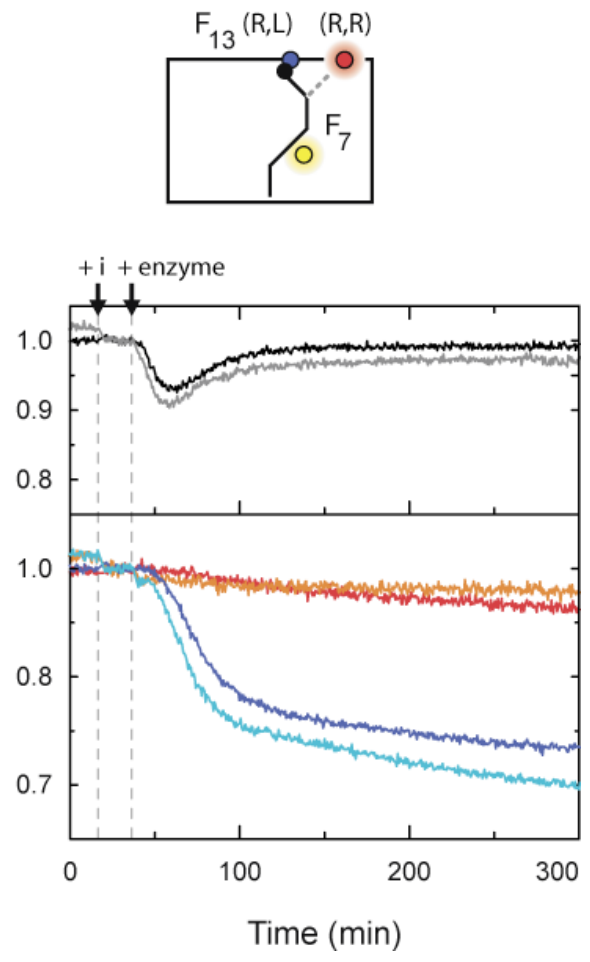

Supplementary Figure 6: Testing additional control domains for the two-layer track. A second set of binding, block and unblock domains was designed for the control stators downstream of nodes 2 and 3 in the two-layer track. These strands were tested independently on the right-hand side only of the two-layer DNA origami track. Block strands for the first junction were omitted during tile assembly. The generic unblock strand was added along with a single instruction strand: a i(-,R), or $\mathbf{b}$ i(-,L). Results are shown for fluorophores $F_{7}(R), F_{13}(R, R)$ and $F_{13}(R, L)$. There is a dip in $F_{7}(R)$ as the motor moves past $S_{7}(R)$, and either $F_{13}(R, R)$ or $F_{13}(R, L)$ is strongly quenched, depending on the instruction strand added, as expected. Leakage is decreased for the twin-block track, in which the block is repeated at stator $\mathrm{S}_{12}$, giving two control stators along each path. This second junction operates similarly to that demonstrated in Figure 2. 

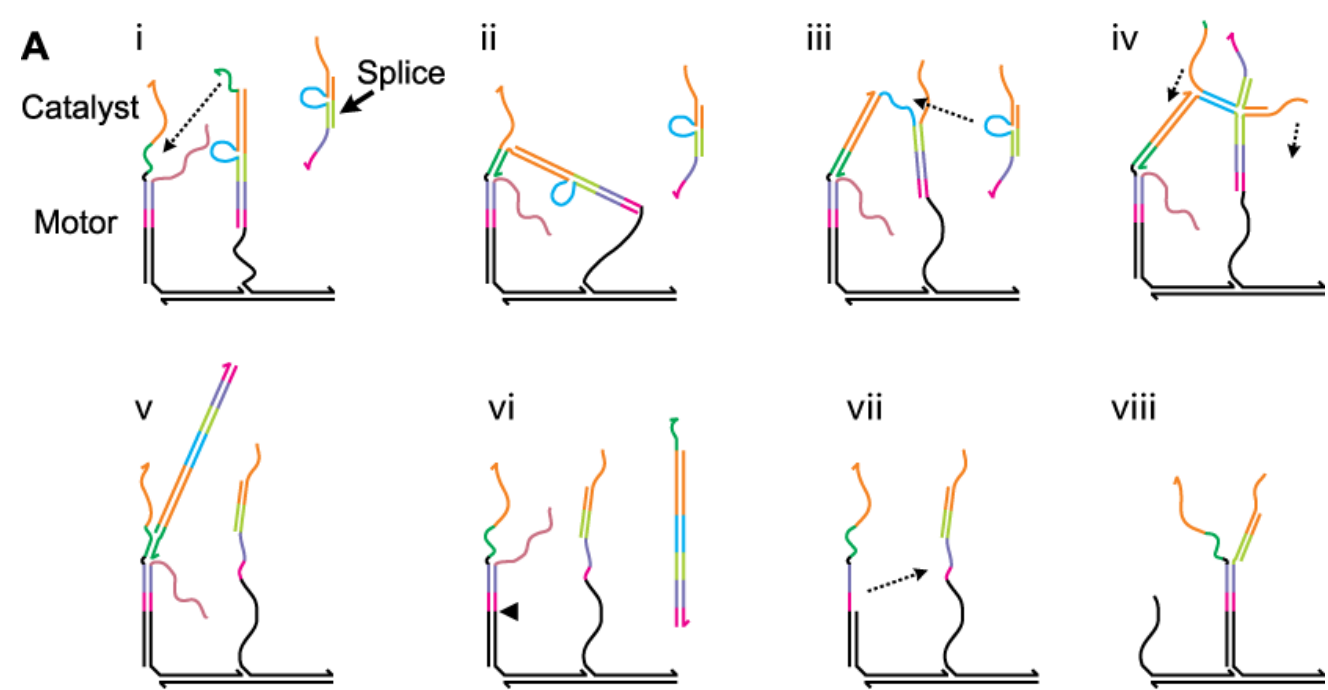

viii
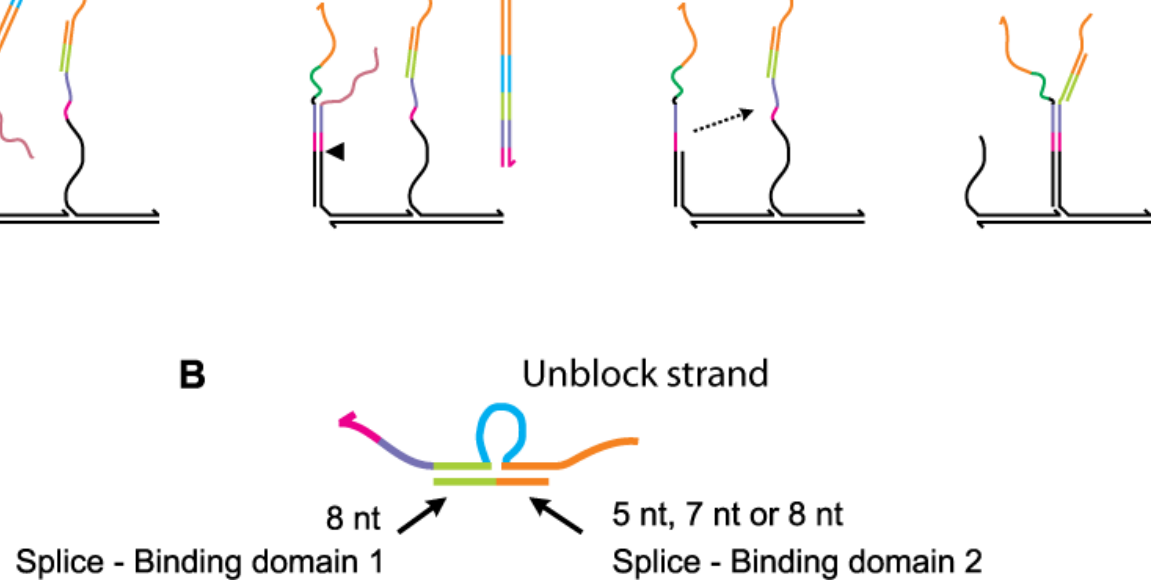

Supplementary Figure 7: Design of the motor-catalysed unblocking system. A Migration of the motor from the unblocked node stator (left) to one of the blocked control stators downstream of the junction (right). The motor is designed to catalyse unblocking of only one of the control stators downstream of the junction. $\mathbf{i}$ The blockstator duplex contains a bulge, or loop, of unpaired bases (blue) and a short ssDNA toehold (dark green). The complementary unblock strand is also held in a loop by a short splice strand (orange + light green). ii-iii The motor carries a catalyst sequence, which is complementary to the toehold and half of the binding domain of the block strand (dark green + orange). This forms a complex with the blocked stator, opening the loop in the block strand (blue). iv-v The unblock strand interacts with the open loop, forming a 4-arm Holliday junction. Migration of the junction removes the block strand from the stator and replaces it with the splice strand. vi The block-unblock duplex is bound to the motor strand by $6 \mathrm{nt}$. This will dissociate, returning the motor strand to its original configuration (cf. i). vi-viii Following enzymatic cleavage of the node stator (black triangle), the motor steps forward onto the unblocked downstream stator. No significant difference in behaviour is expected if cleavage occurs before the unblocking process. B Details of the splice-unblock complex. 
a Motor (L):

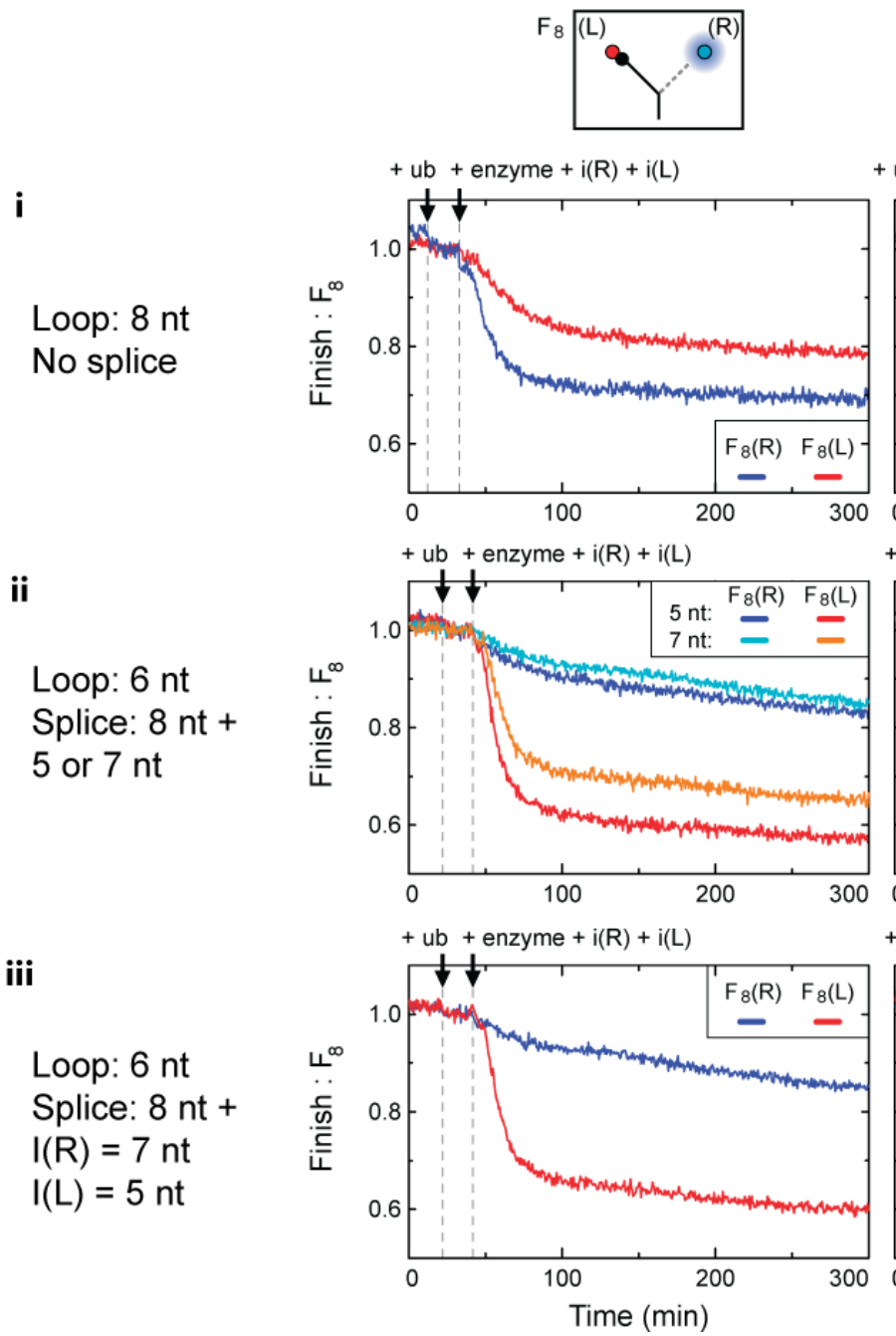

b $\operatorname{Motor}(\mathrm{R})$ :
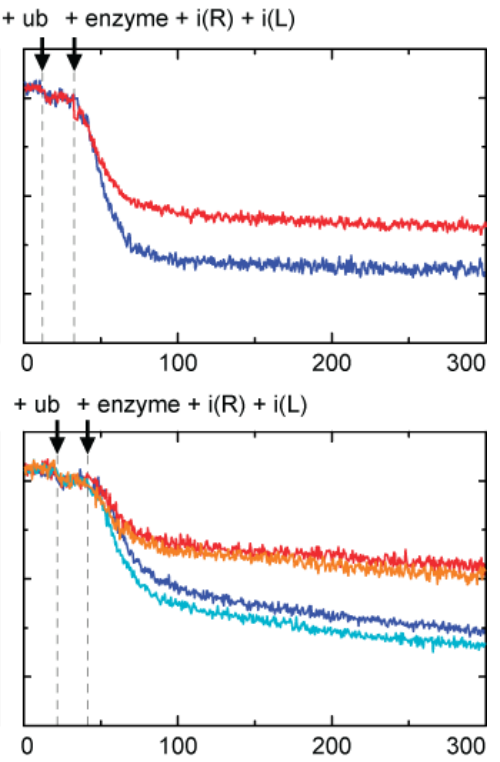

$+u b+$ enzyme $+i(R)+i(L)$

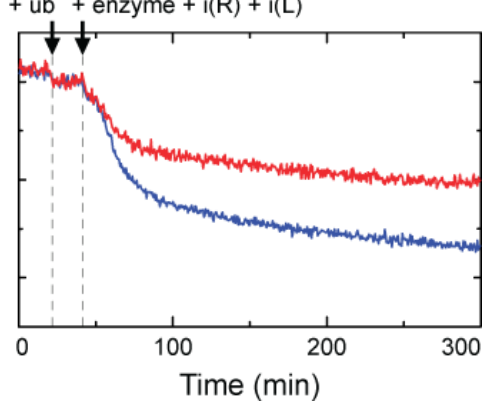

Supplementary Figure 8: Motor-catalysed system: additional fluorescence results. A number of different loop and splice lengths were compared. i Without the splice strand no directional bias between the two motor types was observed. ii A strong bias was observed with 6-nt loops and either 5-nt or 7-nt lengths for the second neck domain of the splice strand. iii The strongest bias was observed with the combination of $\mathrm{i}(\mathrm{R})+(8+7)$-nt splice, and $\mathrm{i}(\mathrm{L})+(8+5)$-nt splice. 


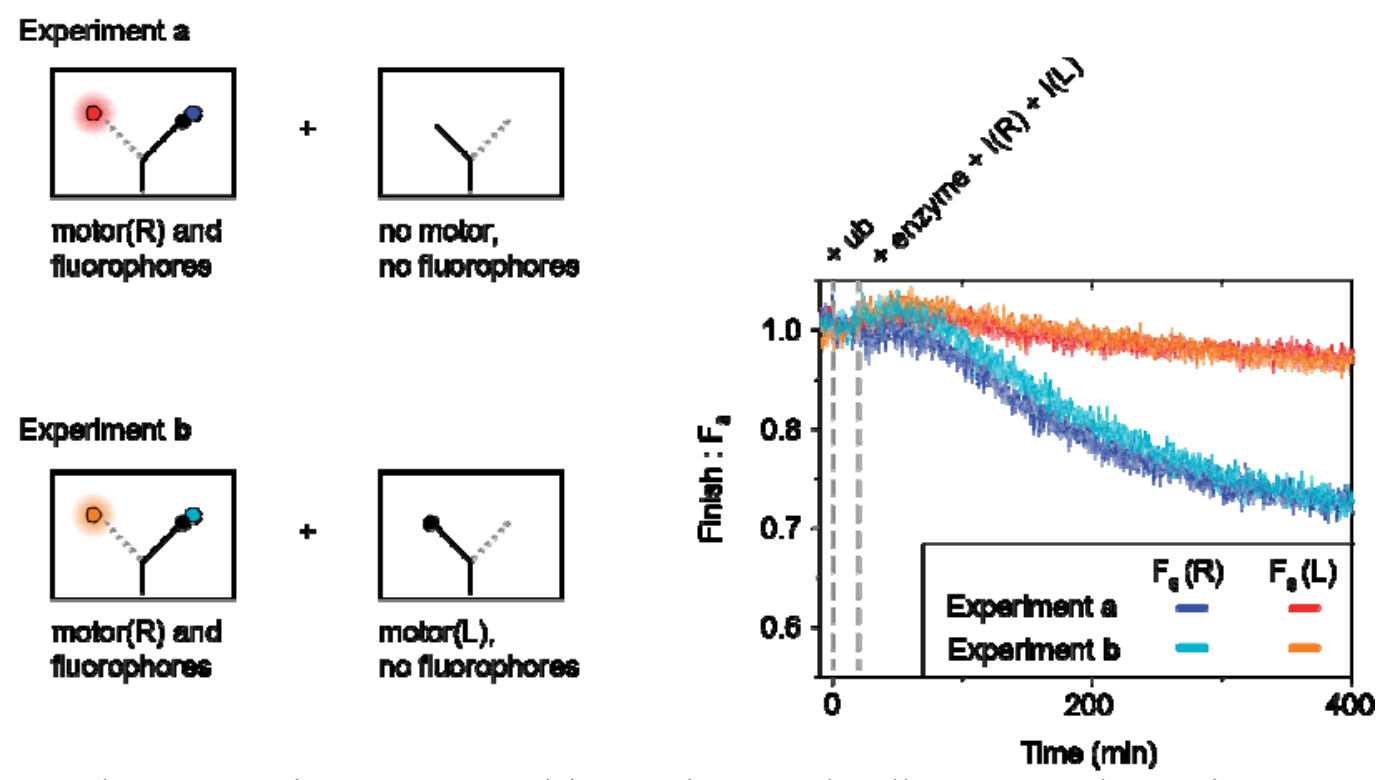

Supplementary Figure 9: Internal instructions act locally. A control experiment was performed to investigate whether the instructions carried by a motor act locally. Two types of tile were mixed: one tile was loaded with motor(R) and labeled with fluorophores to report the arrival of the motor at the left and right branches of a single-layer track; the other tile, with no fluorophores, was either left empty (Experiment a) or loaded with motor(L) (Experiment b). If the instruction carried by a motor does not act locally then motor(L) on the unlabeled tile would unlock the lefthand branch of the labeled tile and reduce or remove the designed bias toward the right-hand branch of the labeled tile. However, a similar bias was observed in both the presence and absence of motor(L) on the unlabelled tile demonstrating that the instructions carried by a motor act locally. 

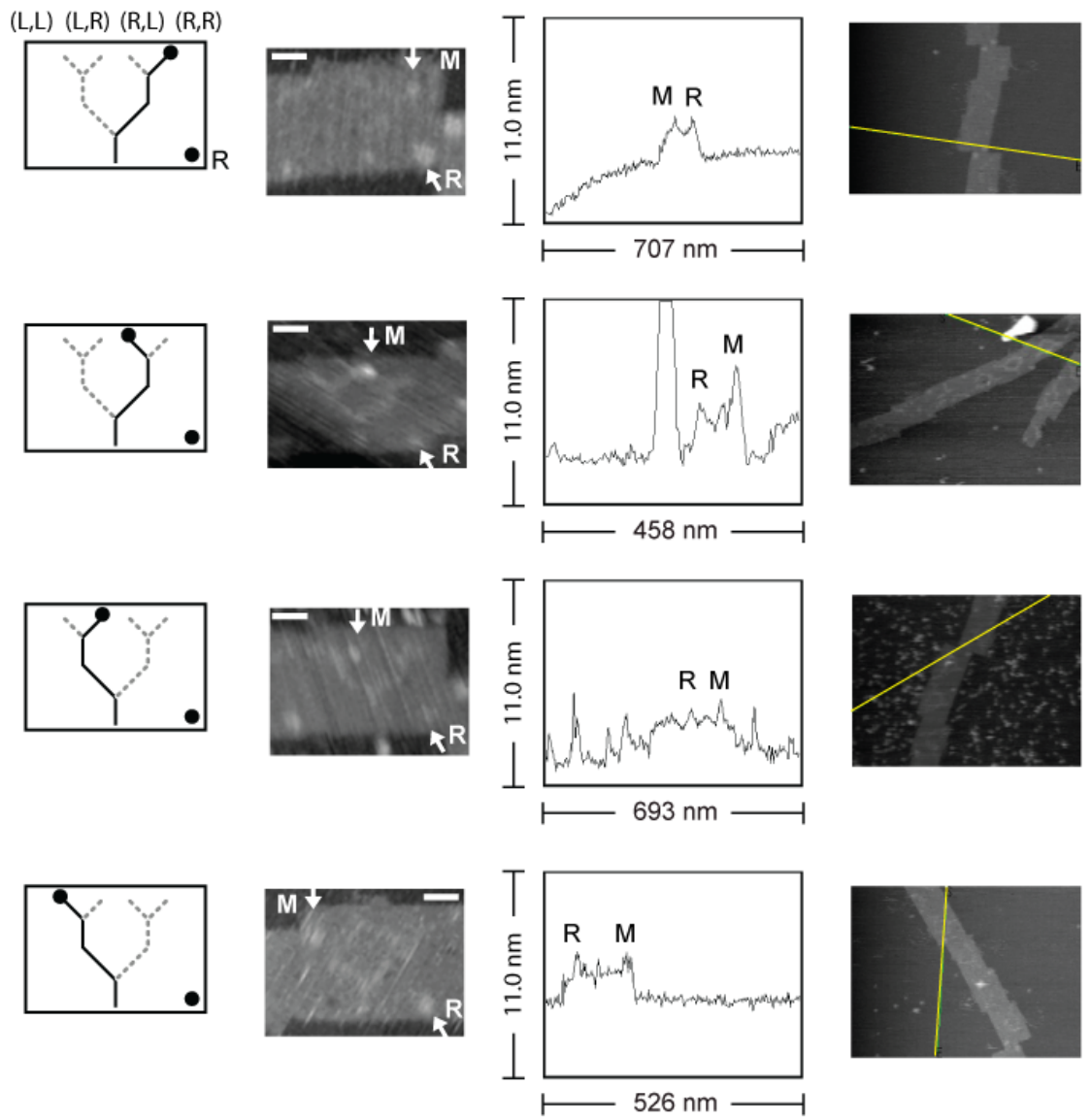

Supplementary Figure 10: Height profiles for AFM data in Figure 3. The height profiles (centre) corresponds to the yellow lines plotted on the expanded images (right). Peaks corresponding to the motor and reference marker are indicated. 


\section{Supplementary Tables:}

\begin{tabular}{|c|c|}
\hline Motor & CGATGTAGTTGGGCTGAGGTTCC \\
\hline S1-i & GGAACCTCAGCCCAACTAACATCGTTTGATATATTAACAACCATCGCCCACCAAGAGAA \\
\hline S2 & TCTTGTACCGTTTGCAGACTGGAACCTCAGCCCAACTAACATTTTGTATCATCGAAACAAAGTACAACCGCCGCCA \\
\hline S3 & TCTTGTACCGTTGCAGACTGGAACCTCAGCCCAACTAACATTTTTGAGATGGCAGAACGAGTAGTAACATTACCA \\
\hline S4 & TCTTGTACCGTTGGAGACTGGAACCTCAGCCCAACTAACATTTTAGGCTTTTACGATAAAAACCAAAATTAAGAC \\
\hline S5 (R) & ACCATTCATACTCTATCCCCGGAACCTCAGCCCAACTAACATTTTTACAGAGAGTCAAAAATGAAAATAGGAAGCAA \\
\hline S6 (R) & TCTTGTACCGTTTGCAGACTGGAACCTCAGCCCAACTAACATTTTTGTTTAACGAATAACATAAAAACTAATAACG \\
\hline$S 7(R)$ & TCTTGTACCGTTTGCAGACTGGAACCTCAGCCCAACTAACATTTTGGCTTATCGCACTCATCGAGAACCCGACAAA \\
\hline S8f $(R)$ & TCTTGTACCGTTTGCAGACTGGAACTTCAGCCCAACTAACATTTTCAAGTACCCGGTATTCTAAGAACGCCATATTA \\
\hline S5 (L) & GCAATCTCGTCACGCCTGTAGGAACCTCAGCCCAACTAACATTTTCGAGCTTCAGGTCAGGATTAGAGGCTATATT \\
\hline S6 (L) & TCTTGTACCGTTTGCAGACTGGAACCTCAGCCCAACTAACATTTTTAATTGCTACTTCAAATATCGCGCCAGAGGG \\
\hline S7 ( L) & TCTTGTACCGTTTGCAGACTGGAACCTCAGCCCAACTAACATTTTGCAAATGGCATACAGGCAAGGCAAGAGTAATG \\
\hline S8f $(L)$ & TCTTGTACCGTTGCAGACTGGAACTTCAGCCCAACTAACATTTTCAAAATTAAGTTTGACCATTAGATCATTTTT \\
\hline
\end{tabular}

Staple strands modified for two-layer track (Fig. S2)

S8 (R) TCTTGTACCGTTTGCAGACTGGAACCTCAGCCCAACTAACATTTTCAAGTACCCGGTATTCTAAGAACGCCATATTA

S9 (R) TCTTGTACCGTTTGCAGACTGGAACTCAGCCCAACTAACATTTTTTAATTGATAATTCTGTCCAGACTATTAAAC

S10 (R) TCTTGTACCGTTTGCAGACTGGAACCTCAGCCCAACTAACATTTTCTACCTTTTATATTTTAGTTAATTAGTAGGGC

S11 (R, R) AGCTAAATCTCGGCCACGTAGGACCTCAGCCCAACTAACATTTTGTACATAATTGCTTTGAATACCAAAAGGGTTA

S12 (R, R) TCTTGTACCGTTTGCAGACTGGAACCTCAGCCCAACTAACATTTTTCGCCTGAATCAATATATGTGAGAGTCAATA

S13f $(R, R)$ TCTTGTACCGTTTGCAGACTGGAACTTCAGCCCAACTAACATTTTAAACAGAATTAAATCCTTTGCCCAATAGATT

S11 $(R, L)$ ACTTATTACTCTATGAAGACGGAACCTCAGCCCAACTAACATTTTTTCATTTGGAGGCGAATTATTCATGATTGTT

S12 (R, L) TCTTGTACCGTTTGCAGACTGGAACCTCAGCCCAACTAACATTTTACCTGAGCACAAAATTAATTACATGGTTGGGT

$\operatorname{S13f}(R, L) \quad$ TCTTGTACCGTTTGCAGACTGGAACTTCAGCCCAACTAACATTTTATGGCAATATCATATTCCTGATTCATAAAGT

S8 (L) TCTTGTACCGTTTGCAGACTGGAACCTCAGCCCAACTAACATTTT CAAAATTAAGTTTGACCATTAGATCATTTTT

S9(L) TCTTGTACCGTTTGCAGACTGGAACCTCAGCCCAACTAACATTTTAAGGCTATTTTAAATGCAATGCCTAGAATTAG

S10 (L) TCTTGTACCGTTTGCAGACTGGACCTCAGCCCAACTAACATTTTTGAGCGAGAGCAAATATTTAAATGATCTACA

S11 $(\mathrm{L}, 1)$ AGCTAAATCTCGGCACGTAGGAACCTCAGCCCAACTAACATTTTCCAGCTTTGCTATTACGCCAGCTATAGCTGT

S12 ( L R R TCTTGTACCGTTTGCAGACTGGAACCTCAGCCCAACTAACATTTTGCCTCTTCCCGGCACCGCTTCTGGTCTGGCCT

S13f $(\mathrm{L}, \mathrm{R})$ TCTTGTACCGTTTGCAGACTGGAACTTCAGCCCAACTAACATTTTTCCACACATGGGGTGCCTAATGATGCCCCAG

S11 (L,L) ACTTATTACTCTATGAAGACGGACCTCAGCCCAACTAACATTTTCAGTATCGGCTGCAAGGCGATTAGGGTACCG

S12 (L,L) TCTTGTACCGTTTGCAGACTGGAACTCAGCCCAACTAACATTTTAACGCCAGCTGCCAGTTTGAGGTCTCCGTG

S13f $(\mathrm{L}, \mathrm{L})$ TCTTGTACCGTTTGCAGACTGGAATTCAGCCCAACTAACATTTTCAGGTCGAGAATCGGCCAACGCGGGTGGTTT

\begin{tabular}{|c|c|}
\hline General-B & GGTTCCAGTCTGCAAACGGTACAAGAAAGTCGCTTCGCACA \\
\hline General-U & TGTGCGAAGCGACTTTCTTGTACCGTTTGCAGACTGGAACC \\
\hline$i(R,-)-B$ & CTGAGGTTCCGGGGATAGAGTATGAATGGTCCACCTTAAAAATGC \\
\hline$i(R,-)-U$ & GCATTTTTAAGGTGGACCATTCATACTCTATCCCCGGAACCTCAG \\
\hline$i(L,-)-B$ & CTGAGGTTCCTACAGGCGTGACGAGATTGCCGATCAAAGCAGTGC \\
\hline$i(L,-)-U$ & GCACTGCTTTGATCGGCAATCTCGTCACGCCTGTAGGAACCTCAG \\
\hline$i(-, R)-B$ & CTGAGGTTC \\
\hline$i(-, R)-U$ & CGCGGACACAAGGGG GCTAAATCTCGGCCACGTAGAACCTCAG \\
\hline$i(-, L)-B$ & CTGAGGTTCCGTCTTCATAGAGTAATAAGTGACACCCTGGTCGAA \\
\hline$i(-, L)-U$ & TTCGACCAGGGTGTCACTTATTACTCTATGAAGACGGAACCTCAG \\
\hline
\end{tabular}


Supplementary Table 1. DNA sequences for modified staples, motor and track strands. Unmodified staple sequences are as in [14]. 


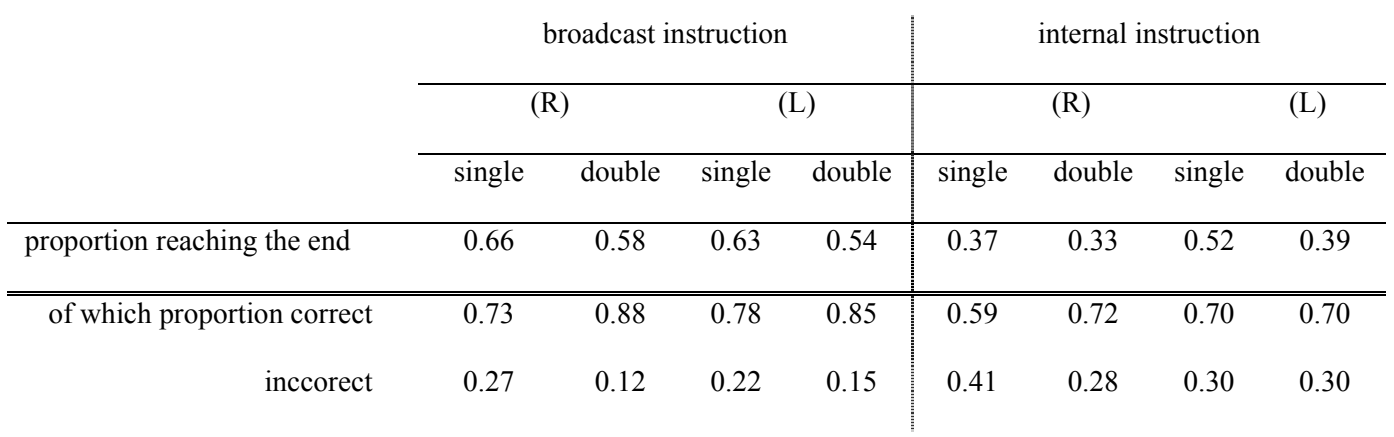

Supplementary Table 2. Bias observed for single-layer tracks. The reliability with which motors interpret instructions on single-layer tracks was deduced from normalized fluorescence signal, $\mathrm{F}$ at $\mathrm{t}=200 \mathrm{~min}$, in Figures 2 and 4 of the accompanying manuscript. The maximum ratio of motor:labeled track is $0.855: 1$ (the ratio of motor: $S_{1}$ is $0.95: 1$ and motor- $S_{1}$ is added at $0.9 \times$ track concentration). We estimate the proportion of motors that reach the end of a specific branch as (1$\mathrm{F}) / 0.855$ where $\mathrm{F}$ is the normalized fluorescence signal from the corresponding fluorophore. This assumes $100 \%$ loading efficiency and is therefore a lower limit. Of the motors that reach the end of the track, the proportion that reach the programmed destination is reported as 'correct', the remainder is reported as 'incorrect'.

\begin{tabular}{rlcccc} 
& predicted & $(\mathrm{R}, \mathrm{R})$ & $(\mathrm{R}, \mathrm{L})$ & $(\mathrm{L}, \mathrm{R})$ & $(\mathrm{L}, \mathrm{L})$ \\
\hline proportion reaching the end & & 0.33 & 0.40 & 0.22 & 0.33 \\
\hline \hline $\begin{array}{r}\text { of which proportion } \\
\text { correct,correct }\end{array}$ & 0.76 & 0.70 & 0.65 & 0.55 & 0.76 \\
correct,incorrect & 0.11 & 0.16 & 0.06 & 0.20 & 0.00 \\
incorrect,correct & 0.11 & 0.07 & 0.20 & 0.22 & 0.22 \\
incorrect,incorrect & 0.02 & 0.07 & 0.09 & 0.02 & 0.02
\end{tabular}

Supplementary Table 3. Predicted and observed bias for two-layer tracks (broadcast instructions). The reliability with which motors interpret instructions on two-layer tracks was deduced from fluorescence signals at $t=200 \mathrm{~min}$ in Figure 3 of the accompanying manuscript. Of all the motors that reach the end of the track, the proportion that take the correct branch at both nodes is reported as 'correct,correct', those that take the incorrect branch at the second node are reported as 'correct,incorrect', etc. The predicted distribution of outcomes on two-layer tracks assumes that the probability, $p$, of taking a correct decision is the same for a node in the first layer as it is for a node in second layer and that $p=0.87$ (Supplementary Table 2 shows a bias toward of 0.88 and 0.85 for broadcast instructions (L) and (R) with double-block tracks). 